\title{
Travail décent et modernisation du droit du travail
}

Decent work and labour market modernisation

\section{Michèle Bonnechère}

\section{(2) OpenEdition}

12 Journals

Édition électronique

URL : http://journals.openedition.org/travailemploi/2324

DOI : 10.4000/travailemploi.2324

ISSN : 1775-416X

Éditeur

DARES - Ministère du Travail

\section{Édition imprimée}

Date de publication : 17 mars 2008

Pagination : 91-101

ISSN : 0224-4365

\section{Référence électronique}

Michèle Bonnechère, «Travail décent et modernisation du droit du travail », Travail et Emploi [En ligne],

113 | janvier-avril 2008, mis en ligne le 17 février 2011, consulté le 19 avril 2019. URL : http://

journals.openedition.org/travailemploi/2324; DOI : 10.4000/travailemploi.2324 


\title{
Travail décent et « modernisation » du droit du travail
}

\author{
Michèle Bonnechère (*)
}

\begin{abstract}
L'article analyse les concepts de flexibilité et sécurité sous-jacents aux projets de réforme du marché du travail débattus en France et met en cause la vision des rapports entre économie et droit du travail qui les sous-tend. Il souligne les limites d'une pensée qui serait fondée exclusivement sur l'efficacité économique du droit et n'intégrerait pas, notamment, les valeurs et les acquis portés par le droit international et européen. L'auteure se place sur le terrain de la qualité des emplois et des principes développés par l'OIT sur le travail décent; elle oppose la notion de droits fondamentaux aux conceptions économistes qui priment dans les discussions actuelles. Elle insiste notamment sur le caractère irréversible de certains principes ou règles du droit du travail, tels que le droit fondamental des travailleurs à l'information-consultation ou les obligations d'adaptation et de reclassement préalables à tout licenciement économique et détaille les principaux obstacles juridiques à la flexicurité.
\end{abstract}

L'idée qu'un point de rupture est atteint par l'ordre public de protection et que seuls des assouplissements du droit du travail peuvent assurer davantage d'emplois est ancienne: on peut rappeler la longue négociation collective sur «la flexibilité» (28 mai-6 décembre 1984) et son échec. Étaient en cause déjà l'accès aux contrats à durée limitée, les conditions du licenciement économique, les seuils d'effectifs, l'aménagement du temps de travail. De simples garanties de procédure et des engagements sans consistance ne furent pas jugés suffisants pour justifier la mise en cause de certaines dispositions du Code du travail. Vingt ans après, une partie de la doctrine juridique exprime toujours ses doutes face à l'idée que les problèmes d'emploi tiennent au droit du travail et que celui-ci devrait restituer à l'économie la liberté de s'autoréguler.

Le thème de la «flexicurité» diffère du débat qui avait lieu il y a vingt ans en introduisant la fameuse recherche d'équilibre et de compensations pour les salariés, dans un contexte où le contrat de travail assure de moins en moins un statut professionnel stable. Si on la prend au sérieux, cette quête de solutions nouvelles doit placer le social au cœur de l'économique. «Dans une économie flexible désireuse de maximiser sa croissance économique et son niveau d'emploi, les préoccupations relatives au

(*) Professeur de droit du travail à l'université d'Évry, Val d'Essonne. Centre Léon Duguit. michele.bonnechere@univevry.fr potentiel humain deviennent premières » relevait le rapport de la commission Supiot ( $c f$. SUPIOT, 1999, spécialement, chap. VII, p. 268).

Il faut écrire clairement que l'optique du «travail décent» ne s'inscrit pas dans la perspective d'une recherche d'«efficacité» du droit du travail (pour qui ?), mais introduit la référence à une valeur fondatrice de ce droit, proclamée par la déclaration de Philadelphie, base constitutionnelle de l'OIT en 1944: «le travail n'est pas une marchandise». Valeur réaffirmée à travers ce concept de travail décent depuis le rapport SomAVIA (1999)(1). Les droits du travail sont interpellés sur le terrain de la qualité de l'emploi en rapport avec les conditions de vie, avec une exigence d'autonomie et de «capacité»(2) des personnes (au travail ou en recherche d'emploi), de droits collectifs aussi. Ceci s'accorde bien avec les analyses économiques mettant au premier plan les coûts humains du travail et du développement (BARTOLI, 1991; 1996), mais est incompatible avec des systèmes envisageant les hommes comme des moyens devant être adaptés aux exigences du marché. L'on pense à l'ambiguité

(1) Le travail décent est selon l'OIT «l'activité qui assure liberté, sécurité, dignité et équité à qui le réalise, en fonction des critères individuels et collectifs de l'être humain concerné».

(2) $\mathrm{Au}$ sens où le rapport précité, reprenant une thèse de R. Salais, et des idées développées par A. Sen, parle de «capacités au travail flexible», opposant "sécurité face à l'aléa» et protection contre celui-ci, et soulignant: «il n'y a de convention de confiance dans la situation de travail que si l'individu est assuré que les projets de vie qui lui tiennent à cœur demeurent réalisables» (op. cit., p. 278). 
du concept «d'employabilité» ou à l'expression «capital humain».

Dans un contexte où l'objectif affiché est de moderniser le droit du travail (COMMISSION EUROPÉENNE, 2006a), cette étude voudrait d'abord souligner l'importance d'une réflexion sur les concepts utilisés et sur la vision des rapports entre l'économie et le droit du travail qu'ils véhiculent, puis rappeler certains points irréversibles de l'évolution du droit du travail.

\section{Enjeux conceptuels}

La définition des concepts de «flexibilité» et de «sécurité» doit être recherchée d'autant plus qu'elle renvoie à des perspectives divergentes voire contradictoires. La perspective du «travail décent» conduit à opposer, à une approche simpliste de la «flexicurité», situant le besoin de souplesse du côté de l'entreprise et les sécurités du salarié comme des limites, une «flexicurité » construite sur de nouveaux droits pour les salariés. Ceux-ci devraient bénéficier de formes de flexibilité, par exemple dans la gestion des temps. Et inversement, l'intérêt de l'entreprise peut être de rechercher la stabilité. Le concept même de travail décent présente aussi un caractère «révolutionnaire» par la relation qu'implique ce «but fondamental» (3) entre éthique sociale, économie et ordre juridique.

\section{Définitions (essai)}

De quelle flexibilité parle-t-on? L'idée générale de souplesse, recherchée par le patronat dans la «gestion de la main-d'œuvre» est mise en œuvre à travers des techniques diversifiées: individualisation des relations de travail, diversification des contrats, articulation nouvelle des normes collectives, «décentralisation» des règles.

a) Appliquée aux sources du droit du travail, la flexibilité pose le problème du rôle respectif de la loi, des conventions et accords collectifs, des règles impératives et des accords «dérogatoires». Dans la théorie des sources du droit du travail, la hiérarchie des normes est établie en fonction de leur teneur, non de leur nature. L'ordre public au sens du droit $\mathrm{du}$ travail ne fait pas obstacle aux dispositions conventionnelles plus favorables aux salariés que celles de la loi(4). L'article L. 132-4 du Code du travail, dont se dégage un principe général du droit,

(3) Discours du directeur général du Bureau international du travail, J.A. Somavia, en 1999.

(4) À ce jour aucune réforme n'a touché à l'article L. 1324 du Code du travail: «La convention et l'accord collectif de travail peuvent comporter des dispositions plus favorables aux salariés que celles des lois et règlements en vigueur. Ils ne peuvent déroger aux dispositions d'ordre public de ces lois et règlements». mais pas un principe constitutionnel(5), exprime la positivité du principe de faveur dans les rapports entre la loi et l'accord collectif. Selon le Conseil constitutionnel, seule une habilitation législative expresse et précise peut autoriser la conclusion d'accords dérogatoires (dont le champ a été considérablement élargi par les dernières réformes, touchant notamment à la consultation du comité d'entreprise sur les licenciements).

Par contre, le principe de faveur ne domine plus l'articulation des accords collectifs entre eux depuis la loi du 4 mai 2004. Sauf clause expresse contraire de la convention de branche (ou de l'accord ayant un champ plus large), l'accord d'entreprise peut comporter des dispositions différentes. Le seul silence de la convention de branche fait de celle-ci une norme supplétive. La mutation est importante puisque le mécanisme de l'extension des conventions collectives s'en trouve par suite affaibli (NADAL, 2005) et le rôle traditionnel de régulation de la concurrence remis en cause pour la convention de branche.

Dans le même temps se multiplient les hypothèses où l'accord d'entreprise est mis en mesure, à égalité avec la convention de branche, de "déroger» (6) à la réglementation légale du travail et de l'aménager: durée du travail, taux des heures supplémentaires, mais aussi droits des travailleurs précaires et même formation de membres des CHSCT(7).

b) Appliquée au contrat de travail, la flexibilité renvoie au «travail différencié», à la place assignée aux contrats précaires car ne procurant pas un véritable emploi : contrats à durée déterminée, contrats temporaires, contrats à temps partiel. De fait, le Code du travail offre à l'employeur, par ces différents types de contrats de travail, de nombreux modes d'ajustement des effectifs aux variations de la conjoncture. La logique de la jurisprudence des éventuelles requalifications des contrats précaires

(5) Dans son avis du 22 mars 1973 (Droit ouvrier, 1973 190) le Conseil d'État a dégagé de l'article L. 132-4 (anciennement article 31a) comme principe général du droit, la signification spécifique de l'ordre public en droit du travail, ce qu'il a confirmé ensuite (not., CE, 27 juillet 2001, RJS 1/02, no 107 et chron. F. Bocquillon). De son côté le Conseil constitutionnel n'y voit pas un «principe fondamental garanti par les lois de la République», mais un principe fondamental du droit du travail au sens de l'article 34 de la Constitution, ce qui autorise le législateur à en réduire le champ d'application. (notamment CC, 29 avril 2004; Déc. 2004-494 DC). Quant à la Cour de cassation, elle a fait référence au "principe fondamental, en droit du travail, selon lequel la situation des salariés doit être régie, en cas de conflit de normes, par celle qui leur est la plus favorable» (Soc., 17 juillet 1996, Grands arrêts du droit du travail, Dalloz, 2004, $\left.\mathrm{n}^{\circ} 173\right)$.

(6) L'expression «accords dérogatoires» n'est adéquate que si la convention ou l'accord est en présence d'une norme impérative. Elle convient donc aux hypothèses où l'accord ou la convention peuvent adapter la loi, elle est impropre, malgré la pratique des bilans officiels et de nombreux commentaires, lorsque l'accord d'entreprise diverge de la convention de branche, celle-ci n'ayant plus d'impérativité de principe.

(7) Voir par exemple articles L. 124-4-4 et L. 236-10. 
en contrats à durée indéterminée n'est pas toujours simple à saisir: après avoir facilité le recours aux contrats à durée déterminée «d'usage» (8) par une nouvelle interprétation des textes, la chambre sociale de la Cour de cassation a invité les juges du fond à vérifier que «l'accroissement temporaire d'activité», hypothèse de recours légal aux contrats à durée déterminée comme aux contrats temporaires, correspondait à autre chose qu'une augmentation constante de la production d'une entreprise(9). Puis elle s'est alignée sur la jurisprudence communautaire en limitant les contrats d'usage successifs à l'existence de raisons objectives liées au caractère effectivement temporaire de l'emploi(10). Mais peuvent être également remises en cause les règles applicables au contrat à durée indéterminée en matière de licenciement, ainsi que le montre le régime du «contrat nouvelles embauches», dont la non-conformité aux normes internationales est établie (voir infra). De même les propositions visant à substituer des incitations financières à la nécessité d'une cause réelle et sérieuse et à son contrôle par le juge se heurtent-elles à des règles fondamentales (voir infra).

c) Appliquée à la durée du travail, la flexibilité correspond à l'introduction de nombreuses souplesses dans la définition et la gestion des temps de travail. Depuis 1982, l'aménagement du temps de travail est le domaine de prédilection des accords collectifs dérogatoires(11). L'exemple des conventions de forfait montre que l'équilibre auquel renvoie le vocable de «flexicurité» est loin d'être atteint dans les solutions en vigueur. Selon l'article L. 212-15-3 du Code du travail, la durée du travail des cadres «autonomes» peut être fixée par des conventions individuelles de forfait en jours établies sur la base de la semaine, du mois ou de l'année, dès lors que ce mécanisme est prévu par une convention ou un accord collectifs étendus ou un accord d'entreprise ou d'établissement. Ceci

(8) Soc., 26 novembre 2003, quatre arrêts, Droit ouvrier, 2004217, note M. Henry et M. F. Bied-Charreton; ces arrêts opèrent un revirement de jurisprudence: les juges n'ont plus à rechercher si le salarié occupe durablement un poste lié à l'activité normale et permanente de l'entreprise (article L. 122-1 du Code du travail) s'ils constatent que, pour l'emploi considéré, il est effectivement d'usage constant de ne pas recourir au contrat à durée indéterminée (sauf si une convention collective prévoyait le contraire)

(9) Soc., 21 janvier 2004, Bull. V. no 27: «...le recours à des salariés intérimaires ne peut être autorisé que pour les besoins d'une ou plusieurs tâches résultant du seul accroissement temporaire de l'activité de l'entreprise, notamment en cas de variations cycliques de production, sans qu'il soit nécessaire ni que cet accroissement présente un caractère exceptionnel, ni que le salarié recruté soit affecté à la réalisation même de ces tâches ».

(10) Soc., 23 janvier 2008, $\mathrm{n}^{\circ} 06-44197$ et $\mathrm{n}^{\circ} 06-43040$, dans le prolongement de CJCE 4 juillet 2006, Aff. C212/70, Adeneler, cité note 30 infra.

(11) La doctrine parle d'un affaiblissement du modèle légal du temps de travail (Pélissier, Supiot, Jeammaud, Précis Dalloz, Droit du travail, 23e édition). concerne aussi (depuis la loi sur les PME du 2 août 2005) les salariés non cadres dont la durée du travail ne peut être prédéterminée et qui disposent d'une réelle autonomie dans l'organisation de leur emploi $\mathrm{du}$ temps pour l'exercice des responsabilités qui leur sont confiées. Certains observateurs ont noté que la souplesse du cadre conventionnel n'a pas jusqu'à présent abouti «à la mise en place de sécurités originales (aménagement des carrières, formation, évolutions, acquisition et reconnaissance des compétences)» (Duclos, Kerbouc'H, 2006) dans les accords collectifs. On peut penser qu'il est nécessaire de corriger l'insuffisante orientation par la loi de la négociation collective en matière de durée du travail (l'équilibre des parties étant loin d'être assuré). Le Comité européen des droits sociaux a précisément conclu à la violation par la législation française de la Charte sociale européenne(12), le respect des durées maximales journalières et hebdomadaires du travail n'étant pas assuré par la seule fixation d'une limite en jours (218) sur l'année.

d) Appliquée au salaire, la flexibilité peut concerner ses modes de fixation ou son «individualisation». Les conventions de forfait évoquées permettent d'allonger le temps de travail sans rémunération supplémentaire, bien que des mécanismes correcteurs existent dans la jurisprudence(13). Mais diverses clauses dans les contrats de travail tendent à développer la «flexibilité salariale»: clauses de variabilité d'éléments de la rémunération, clauses d'objectifs dont l'obtention est récompensée par des primes. Rien n'empêche la négociation collective d'encadrer de telles clauses, qui pourtant ne relèvent pas du champ de la négociation annuelle obligatoire sur les salaires. Seule la jurisprudence leur a fixé des règles minimales: obligation de fonder les variations sur la base d'éléments ne dépendant pas de la volonté de l'employeur et ne faisant pas supporter le risque d'entreprise par le salarié, condition de faisabilité des objectifs.

Quelle sécurité? Le droit du travail vise en premier lieu la sécurité dans le travail, par les obligations de prévention édictées en droit communau-

(12) Décisions du 16 novembre 2001 et du 12 octobre 2004, RJS 2002.513 et 2005.512, commentaires J. Ph. Lhernould. Le Comité européen des droits sociaux n'est pas une juridiction, mais un comité d'experts qui se prononce soit sur la base des rapports gouvernementaux concernant la mise en œuvre de la Charte sociale européenne, soit dans le cadre d'une procédure de «réclamations collectives» émanant de syndicats ou d'ONG.

La Charte sociale européenne, élaborée dans le cadre du Conseil de l'Europe, affirme par son article 2 le droit à des conditions de travail équitables et l'engagement des parties contractantes «à fixer une durée raisonnable au travail journalier et hebdomadaire».

(13) Exigence d'un accord du salarié, qui ne se présume pas, fixation précise du forfait, ne pouvant être modifié que de l'accord des deux parties... 
taire (14), comme en droit interne à travers la loi et le contrat de travail. L'obligation de sécurité de résultat rattachée au contrat de travail par la Cour de cassation représente une étape essentielle(15).

\section{Prolongement}

Dans la suite du canevas ci-dessus, la réflexion doit également porter sur les aspects suivants:

a) L'équilibre des forces dans la négociation collective et la capacité pour celle-ci de maîtriser l'avenir. Le thème de la sécurité juridique est volontiers abordé à l'occasion des réformes législatives, pour souligner le nécessaire respect des accords par le législateur ou par le juge (16), ou dans les cas de revirements de jurisprudence (17).

Une question très importante devrait lui être rattachée: quelle est la valeur juridique des engagements sur l'emploi dans les accords collectifs?

Le problème est d'abord à poser à partir de l'article L 320-2 du Code du travail, qui impose depuis la loi du 18 janvier 2005 aux entreprises et groupes occupant 300 salariés et plus, une obligation triennale de négocier sur la gestion prévisionnelle de l'emploi et des compétences (GPEC), ainsi que sur l'information-consultation du comité d'entreprise «sur la stratégie de l'entreprise et ses effets prévisibles sur l'emploi ainsi que sur les salaires». L'une des nombreuses ambiguïtés de ce texte novateur concerne la liaison s'y trouvant avec la négociation collective, portant cette fois-ci sur la procédure de licenciement collectif ou même le contenu d'un plan de sauvegarde de l'emploi(18). En effet, la négociation sur la GPEC n'ayant de sens que dans un préalable aux restructurations, n'y a-t-il pas manipulation de la loi sur l'objet de l'obligation de négocier?

(14) C'est la directive-cadre 89/391 du 12 juin 1989 (JOCE du 29 juin 1989), selon laquelle la prévention doit intervenir «à tous les stades de l'activité dans l'entreprise», dont la mise en œuvre a notamment imposé au droit français de prévoir l'obligation d'évaluer les risques.

(15) Soc., 28 février 2002, concl. Benmakhlouf, G.P. 3-5 mai 2002.3; Droit Social, 2002445 , note A. Lyon-Caen; Le Droit ouvrier, 2002 166, note F. Meyer. L'absence de faute n'exonère pas l'employeur: Soc., 21 juin 2006, Le Droit ouvrier, 2006, note F. Saramito.

(16) Selon le Conseil constitutionnel, le législateur ne peut porter atteinte aux conventions légalement conclues sans motif d'intérêt général suffisant (Cons. Const., 13 janvier 2003, DC 2002-465, Rev. contrats déc. 2003.9. n. T. Revet). D'autre part depuis la loi Aubry II, des interventions législatives ont entendu valider des accords collectifs illégaux au moment de leur conclusion (exemple: l'article 45 de la loi du 4 mai 2004 sur le dialogue social).

(17) Rapport Molfessis sur les revirements de jurisprudence remis au premier président de la Cour de cassation en novembre 2004.

(18) L'article L. 320-2 prévoit que la négociation sur la GPEC «peut porter également» sur les matières prévues à l'article L. 320-3, c'est-à-dire notamment les conditions de négociation d'un plan de sauvegarde de l'emploi et même l'anticipation sur son contenu.
Quant à la sécurité que pourraient théoriquement apporter aux salariés les engagements collectifs en matière d'emploi, souvent assortis de la diminution d'avantages salariaux dont ils sont censés être la contrepartie, elle dépend largement des sanctions applicables en cas de non-respect de tels engagements. La jurisprudence reconnait depuis une dizaine d'années un droit à réparation du salarié licencié au mépris d'un engagement de maintien de l'emploi, mais une partie de la doctrine soutient la thèse de la nullité d'un tel licenciement. Afin d'assurer que des solutions négociées soient porteuses de l'équilibre recherché de la «flexicurité», l'efficacité des accords collectifs doit être assurée par de telles solutions, qui sont pourtant loin d'être celles de la Cour de cassation. Sur le plan collectif une autre perspective est celle du recours à la nullité des clauses sur l'emploi imprécises, lorsque la négociation «donnant-donnant» est en fait une pure négociation de concession.

b) Sur la « sécurité de l'emploi »: il s'agit d'abord de la protection contre le licenciement arbitraire et de la garantie d'une stabilité du lien d'emploi (du contrat de travail), mais aussi de la «sécurité des parcours professionnels». Dès l'arrêt Expovit du 25 février 1992, la Cour de cassation, en déduisant de la bonne foi contractuelle, que «l'employeur a le devoir d'assurer l'adaptation de salariés à 'évolution de leurs emplois», a rapproché capacité et sécurité professionnelles. Il faut souligner aussi que si la sécurité de l'emploi est dissociée de la continuation du lien d'emploi (dans la conception du CERC, 2005), la question de la nature du contrat est essentielle, une succession de contrats à durée déterminée ou un contrat hybride sans garanties ayant peu à voir avec la sécurité de l'emploi (voir infra).

c) Dans le domaine de la durée du travail, la sécurité est indissociable du respect des droits fondamentaux du travailleur (voir infra).

d) En matière de salaire, la sécurité résulte du respect du salaire contractuel, du principe d'égalité et d'un niveau de salaire permettant une vie décente.

\section{De "l'efficacité" du droit du travail au travail décent}

En postulant certains droits comme «droits sociaux fondamentaux », l'optique du travail décent (BonNeChÈRE, 2007) s'oppose tant aux approches dites scientifiques ou "positivistes» du droit fondées sur une "neutralité axiologique», qui les conduit à rejeter à l'extérieur du droit toute référence aux valeurs humaines, qu'aux doctrines estimant trouver la source de la règle de droit et sa légitimité dans un calcul d'utilité ou d'efficacité. L'efficacité, qui renvoie à la garantie d'un résultat (à définir) ne doit pas être confondue avec l'effectivité, mesurant la distance entre le fait et le droit.

Le rapport Pour un Code du travail plus efficace de la commission de VIRVILLE (2004) avait pour défaut de prétendre évaluer le droit du travail du point de vue de 
l'efficacité de l'activité économique des entreprises alignant critiques et propositions, sans préciser les critères de cette efficacité recherchée des règles applicables aux rapports de travail. Le premier président de la Cour de Cassation, sensible au courant de l'analyse économique du droit(19), après avoir affirmé que celle-ci rappelait brutalement le droit français à «l'impératif d'efficience», a lui même souligné que «la logique juridique sociale est différente de la logique économique»(20). Le président de la chambre sociale a ensuite réagi vivement à l'idée que les revirements de jurisprudence produisent des effets «fondamentalement anti-économique», selon l'expression du rapport Molfessis qui n'en donne pas de définition (SARGOS, 2005, p. 123). De son côté, l'association Henri Capitant a contesté le postulat de départ dans une étude sur les droits de tradition civiliste(21), ainsi que la méthode des rapports 2004 et 2005 de la Banque mondiale: elle a développé l'idée que «le droit français ne cherche pas l'efficience économique pour elle-même à tout prix et à court terme, mais se veut fondateur d'un modèle de société durable».

Le juriste a sûrement du mal à saisir pleinement les analyses économiques. Mais une chose est sûre: les droits individuels et collectifs des salariés, affirmés progressivement, ne sont ni la contrepartie «sociale» du lien de subordination, ni des éléments permettant au salarié de calculer une «stratégie optimale» dans sa relation de travail et conditionnant son engagement productif(22). L'explicitation du lien de subordination juridique dans le contrat de travail par les tribunaux, qui en ont fait le critère, a fait apparaître la personne du salarié. Tout en opérant une «mise à nu» des rapports de travail, le lien de subordination révèle que le contrat ne concerne pas des choses mais des individus. Comme le courant $\mathrm{du}$ «droit capitaliste du travail» l'a démontré, ce critère est très adéquat au mode de fonctionnement d'une économie fondée sur les pouvoirs du chef d'entreprise. Mais il situe le travailleur comme sujet, et donne en même temps au droit du travail sa finalité: tendre au rétablissement d'un équilibre par l'affirmation des droits de la personne au travail et des droits d'action collective.

Aujourd'hui le concept de travail décent infirme en outre la démarche positiviste d'une partie majo-

(19) L'analyse économique du droit (AED) correspond à une série de thèses doctrinales, partie des États-Unis, estimant nécessaire d'apprécier systématiquement l'incidence économique des règles juridiques et de proposer des méthodes pour ce faire.

(20) G. Canivet, Audience solennelle de rentrée de la Cour de cassation, 7 janvier 2005.

(21) «Les droits de tradition civiliste en question. À propos des rapports Doing business de la Banque mondiale», Association H. Capitant des Amis de la culture juridique française, Société de législation comparée, 2006.

(22) Une approche économique dite institutionnaliste semble situer la liberté du salarié dans la relation de travail par rapport à son engagement productif, qu'elle conditionnerait: F. EYMARDDuverNAY, 2004, p. 812-815; 2005, p. 87 et suiv. ritaire de la doctrine juridique. Celle-ci situe audehors de la science du droit, dans la logique de Hans Kelsen, les principes d'éthique et de justice. «La tâche de la science du droit, c'est la description du droit positif, la description des normes en vigueur et rien de plus» a écrit M. TROPER (1990) dans une réponse fameuse à l'article de D. LOCHAK (1990) analysant le rôle de la doctrine sous Vichy (23).

L'approche positiviste, qui récuse la démarche «prescriptrice», ne peut guère admettre que des droits soient «postulés». L'optique du travail décent éclaire aussi les divergences entre l'analyse économique dominante et l'«économie multidimensionnelle» de H. Bartoli (le principe d'économicité a pour contenu la recherche de la couverture des besoins du statut humain de la vie) ou l'approche globale prônée par A. Sen. Sur le plan de la stricte technique juridique, la subordination est à la fois ce qui détermine la qualification de contrat de travail et ce qui déclenche le bénéfice des droits du salarié. Ces brèves remarques font entrevoir une conception pouvant être perturbatrice de la «flexicurité» en droit du travail.

\section{La non-réversibilité de certains principes ou règles du droit du travail}

Alors que le recours à l'analyse comparative pour sortir du modèle français est régulièrement conseillé, il serait paradoxal que soient ignorées les normes juridiques universelles ou européennes applicables aux relations de travail dans la recherche de réponses aux défis de la mondialisation en matière d'emploi. Quant à la réflexion sur le rôle du juge, considéré comme excessif ou inadapté par des observateurs qui voudraient réduire les hypothèses et la portée du contrôle juridictionnel, elle ne saurait ignorer que la jurisprudence est une partie essentielle, très construite, du droit du travail français, et que certains de ses apports sont incompressibles.

\section{Exigences des normes internationales et européennes}

Beaucoup l'ont découvert à propos du contrat «nouvelles embauches», il existe des normes internationales du travail auxquelles certaines évolutions des modèles nationaux pourraient donner une nouvelle actualité. Certes, l'efficacité des protections garanties par l'Organisation internationale du travail est contestée, mais sa compétence est reconnue par l'Organisation mondiale du commerce dans les négociations internationales et demeure centrale. Quant au droit social communautaire, malgré son

(23) À noter qu'une minorité d'auteurs se réclament explicitement d'un courant de philosophie du droit, la doctrine «travailliste» étant particulièrement silencieuse sur ce point. 
existence encore en "pointillé», il s'avère actuellement susceptible d'endiguer certaines formes de dérégulation. Ces tendances s'observent tant pour ce qui concerne la nature du contrat de travail et de sa rupture, que pour les licenciements collectifs.

\section{Aspects du régime du contrat de travail}

La convention $\mathrm{n}^{\circ} 158$ de l'OIT concernant la cessation de la relation de travail à l'initiative de l'employeur impose ainsi un «motif valable» pour tout licenciement(24), ce qui n'est guère conciliable avec la substitution d'obligations ou incitations financières à l'exigence d'une cause (réelle et sérieuse) de licenciement. La France a ratifié cette convention en 1982, mais quinze États membres de l'Union européenne ne l'ont pas fait.

Le droit communautaire expliquerait-il cette situation? Au contraire, la Charte des droits fondamentaux de l'Union européenne, dans son article 30 («Protection en cas de licenciement injustifié») précise: «Tout travailleur a droit à une protection contre tout licenciement injustifié, conformément au droit communautaire et aux législations et pratiques nationales $\gg$.

Dans une intéressante décision du 26 octobre 2006, la juridiction communautaire de première instance(25) a estimé devoir prendre en considération «l'existence de standards internationaux visant à indiquer les conditions minimales nécessaires dans un État de droit pour éviter des licenciements abusifs »». L'arrêt vise à cet égard, outre l'article 4 de la convention OIT 158, la Charte sociale européenne adoptée dans le cadre du Conseil de l'Europe, relevant que son article 24 (lequel affirme «le droit des travailleurs à ne pas être licenciés sans motif valable») a servi de source d'inspiration à l'article 30 de la Charte communautaire. Celle-ci, guide pour l'interprétation des lois nationales, bien que non intégrée au Traité sur l'Union européenne, servira sans aucun doute de fondement à une construction jurisprudentielle.

L'applicabilité directe de la Charte sociale européenne, qui a la valeur d'un traité international, a été reconnue par le Conseil d'État(26), avant que la Cour de cassation ne fasse de même pour plusieurs dispositions de la convention 158 précitée(27). Ces textes sont évidemment susceptibles d'interpréta-

(24) Convention 158 concernant la cessation de la relation de travail à l'initiative de l'employeur: «un travailleur ne devra pas être licencié sans qu'il existe un motif valable de licenciement lié à l'aptitude ou à la conduite du travailleur ou fondé sur les nécessités du fonctionnement de l'entreprise, de l'établissement ou du service.»

(25) Le tribunal de première instance statuait en tant que tribunal de la fonction publique dans une affaire de licenciement d'un agent temporaire (TPT, 26 octobre 2006, Aff. F-1/05).

(26) CE, 19 octobre 2005, Droit ouvrier, 2006.75, note G. Koubi; JCP, Ed. Soc., n ${ }^{\circ} 20$ du 8 novembre 2005, obs. R. Vatinet.

(27) Soc., 29 mars 2006, Bull. civ. V. no 131. tion. La convention 158 permet ainsi à un État d'exclure de la règle évoquée les salariés n'ayant pas une certaine ancienneté, pourvu que la durée imposée soit raisonnable. Pour le Conseil d'État, cette condition était remplie par l'ordonnance sur le CNE (pendant deux ans le droit commun du licenciement est écarté), alors que la cour d'appel de Paris a jugé en sens inverse (28). Sur réclamation de Force ouvrière, le Bureau international du travail a tranché dans un avis du 15 novembre 2007, jugeant que l'ordonnance sur le CNE «s'éloigne de manière significative» de l'article 4 de la convention 158, qualifié de pierre angulaire de la norme internationale. Le principe de la motivation, susceptible de contrôle par le juge, s'impose en droit du travail.

Autre proposition phare: le contrat de travail unique (CAHUC, Kramarz, 2005). Le droit communautaire ne va pas dans ce sens. L'accord-cadre conclu entre la CES, l'UNICE et le CEEP sur le travail à durée déterminée, transformé en norme communautaire par la directive du 28 juin 1999, vise les contrats à durée indéterminée comme «la forme générale de relations d'emploi entre employeurs et travailleurs » (29), qui s'affirme donc comme modèle. La norme communautaire considère ensuite que les contrats à durée déterminée répondent dans certaines circonstances aux besoins des employeurs et à ceux des travailleurs. Le droit communautaire impose, outre le principe d'égalité de traitement, un objectif de prévention des abus résultant de contrats de travail à durée déterminée successifs. La CJCE a récemment donné une portée stricte à ces orientations. Selon ses arrêts de juillet et septembre 2006, les États sont libres quant au choix des sanctions du recours abusif aux contrats de travail à durée déterminée (30) (requalification en contrats à durée indéterminée ou autre sanction efficace) mais doivent interdire les successions de CDD non fondées sur des «raisons objectives». Cette dernière notion est entendue en

(28) Après Conseil de prud'hommes de Longjumeau, la cour d'appel de Paris (18 eh., 6 juillet 2007, no 06/06992) a écarté les dispositions de l'ordonnance du 2 août 2005 sur le CNE comme contraires à la convention OIT 158. Le Conseil d'État, le 19 octobre 2005, n'avait pas statué dans le même sens. Le préfet de l'Essonne a soulevé le problème de la compétence de tribunaux judiciaires en la matière, s'appuyant sur la nature administrative de l'ordonnance avant sa ratification parlementaire. La cour d'appel de Paris ayant rejeté le déclinatoire de compétence (le 20 octobre 2006, Droit ouvrier, 2007 132, note I. Meyrat) en distinguant le contrôle de conventionalité effectué par le Conseil de prud'hommes (primauté de la convention internationale sur la loi), le Tribunal des conflits a dû trancher et il l'a fait le 19 mars 2007 en considérant que l'ordonnance du 2 août 2005 n'avait plus la nature d'un acte administratif ayant été ratifiée implicitement par deux lois. L'arrêt rendu le 6 juillet 2007 par la cour d'appel de Paris juge que l'ordonnance relative au CNE contredit la convention OIT $\mathrm{n}^{\circ} 158$, et en écarte les dispositions.

(29) Préambule de l'accord-cadre du 18 mars 1999 sur le travail à durée déterminée.

(30) CJCE, 7 septembre 2006, Aff. C-53/04 et C-180/04, Europe, novembre 2006, com. 319, obs. L. Driguez. 
fonction de circonstances précises et concrètes (31). La Cour de justice souligne que «les contrats à durée indéterminée sont et resteront la forme générale de relations d'emploi, dès lors qu'ils contribuent à la qualité de vie des travailleurs concernés et à l'amélioration de leurs performances». À l'aune des normes évoquées, le contrat de travail unique tel qu'il a été proposé, apparaît bien comme une "chimère»(32), faisant disparaitre tant l'encadrement limitatif des cas de recours au CDD et le minimum de "sécurité» lié au terme fixé, que l'exigence d'une cause réelle et sérieuse du licenciement dans le cadre du CDI.

\section{L'information-consultation, droit fondamental des travailleurs}

Les procédures de licenciement pour motif économique relèvent de directives communautaires dont les premières versions remontent à 1975, sur les licenciements collectifs, et 1977, sur les transferts d'entreprise (33) imposant aux États membres de prévoir dans leur droit interne l'information et la consultation des représentants des travailleurs. Cette obligation pèse sur l'employeur, en droit communautaire, y compris lorsqu'il n'est pas lui-même à l'origine de la décision de restructuration. Elle correspond à un droit reconnu aux travailleurs comme fondamental par les chartes européennes et a été reprise plus largement par la directive "Vilvorde» de 2002(34). Les salariés aspirent à voir prendre en compte des «solutions alternatives», c'est-à-dire des choix économiques moins coûteux en emplois. Ces procédures, avec leurs limites, sont un outil parfois «efficace» du dialogue social. La Cour de Luxembourg a rappelé la dimension régulatrice du droit social communautaire en la matière (35).

(31) «La notion de raisons objectives... doit être entendue comme visant des circonstances précises et concrètes caractérisant une activité déterminée et, partant, de nature à justifier dans ce contexte particulier l'utilisation de contrats de travail à durée déterminée successifs. Ces circonstances peuvent résulter notamment de la nature particulière des tâches pour l'accomplissement desquelles de tels contrats ont été conclus et des caractéristiques inhérentes à celles-ci ou, le cas échéant, de la poursuite d'un objectif légitime de politique sociale d'un État membre.»

(32) Voir Morvan, 2006, 959 (l'auteur est cependant favorable à des solutions du type CNE).

(33) Aujourd'hui: directive $98 / 59$ du 20 juillet 1998, JOCEL, 225 et directive 2001/23 du 12 mars 2001, JOCE, 22 mars 2001.

(34) Directive 2002-14 du 11 mars 2002, JOCE, 23 mars 2002.

(35) La Cour de justice a jugé que les directives précitées faisaient obstacle à ce qu'une législation nationale décide d'exclure, même temporairement, les jeunes travailleurs du calcul des effectifs servant de base à l'établissement des procédures d'information-consultation, à propos de l'ordonnance 2005892 du 2 août 2005 (CJCE, 18 janvier, 2007. Aff. C-385/05).

\section{Le contrôle judiciaire des mobilités et des licenciements économiques, garantie inamovible}

Que l'on parle de «statut professionnel des personnes», de «sécurisation des parcours professionnels», de «droits attachés à la personne», la question de la place et du rôle du contrat de travail est posée. Tout contrat, dans notre système juridique, a des finalités économiques et sociales, et celles du contrat de travail se sont lentement affirmées. Non seulement le juge rétablit le cas échéant la qualification de contrat de travail, mais il a très progressivement remodelé celui-ci, en dégageant des obligations contractuelles, dont la plus retentissante est l'obligation de sécurité de résultat affirmée à propos du dossier de l'amiante.

\section{Travail subordonné et qualification du contrat}

Des stratégies de contournement du salariat sont apparues il y a une quinzaine d'années (CHAUCHARD, 2005). Elles posent au droit du travail une double difficulté : rétablir d'abord les faux travailleurs indépendants dans leurs droits en requalifiant le prétendu contrat de société, contrat de location, contrat d'entreprise, en contrat de travail, mais aussi reconnaître aux travailleurs économiquement dépendants, ou «parasubordonnés», un «socle de droits» pouvant aller du salaire minimum au droit syndical. Dans son Livre vert précité, la Commission européenne évoque à juste titre la «zone grise» du marché du travail qu'il importe de combattre, tout en admettant que la lutte contre le faux travail indépendant relève du plan national. Ceci renvoie en France aux nombreuses actions en requalification dont connaissent les conseils de prud'hommes, notamment dans les secteurs du bâtiment, de l'hôtellerie, des transports. Un principe a été clairement dégagé en jurisprudence: «l'existence d'une relation de travail salarié ne dépend ni de la volonté exprimée par les parties ni de la dénomination qu'elles ont donnée à leur convention, mais des conditions de fait dans lesquelles est exercéel'activité des travailleurs » (36). Malgré les tentatives ponctuelles du législateur à travers une présomption de non-salariat(37), ou la qualification de «gérant mandataire»(38), on peut

(36) Soc. 17 avril 1991, Bull. civ. V. no 200.; Soc., 19 décembre 2000, Labbane, Dr. soc., 2001.227, note A. Jeammaud; Droit ouvrier, 2001.241, note A. de Senga.

(37) L'article L. 120-3 du Code du travail, introduit par la loi du 11 février 1994 dite "Madelin», abrogé par la loi du 19 janvier 2000 (Aubry II) puis rétabli par la loi du $1^{\mathrm{er}}$ août 2003, pose une présomption de non-salariat pour les personnes inscrites au répertoire des métiers, au registre du commerce et des sociétés ou au registre des agents commerciaux. Mais la preuve contraire peut être établie et la jurisprudence s'attache au lien de subordination.

(38) L'article 19 de la loi du 2 août 2005 sur les PME traite du contrat de gérance-mandat, mais le contentieux des requalifications est abondant, notamment dans l'hôtellerie et la téléphonie mobile. 
penser que le pouvoir de requalification du juge continuera à empêcher qu'une prétendue relation commerciale fasse obstacle à l'application du droit du travail.

\section{Les obligations d'adaptation et de reclassement}

L'obligation de reclassement, d'abord reconnue pour les accidentés du travail puis pour les autres victimes d'une décision d'inaptitude médicale à occuper leur poste de travail, a été construite par la jurisprudence en matière de licenciement économique sur le principe de la bonne foi contractuelle et reprise par la loi Jospin de modernisation sociale en 2002. Il ne faut pas oublier que, dès 1969, l'accord national interprofessionnel sur l'emploi imposait des obligations de reclassement pour les «grands licenciements» collectifs, et qu'une partie du droit du licenciement renvoie au préambule de la Constitution, dont découle «l'exigence constitutionnelle» du droit à l'emploi (39). Après avoir rattaché au contrat de travail l'obligation pour l'employeur de prendre en charge l'adaptation du salarié à l'évolution de son emploi (premier jalon de la «sécurité des parcours »), la jurisprudence a dégagé l'obligation contractuelle de reclassement: cette obligation de moyen préalable à toute décision de licenciement économique se superpose à l'obligation d'élaborer un plan de reclassement (proportionnellement aux éléments dont dispose l'entreprise) dans le cadre du plan de sauvegarde de l'emploi, lorsque celuici doit intervenir(40). L'obligation de reclassement, dite «interne», s'étend, pour sa mise en œuvre, à l'entreprise et au groupe. Elle est la conséquence du droit reconnu à l'employeur de résilier le contrat de travail à durée indéterminée pour un motif économique. Le contrôle du juge n'est pas la contrepartie, comme on a pu le dire, des faiblesses françaises en matière de concertation et de négociation: il lui arrive souvent de garantir au contraire le respect des procédures d'information-consultation imposées par la loi et, on l'a vu, par le droit communautaire.

À l'issue d'une longue évolution, la cause juridique du licenciement économique est devenue l'impossibilité de reclassement établie par l'employeur, lequel n'est donc pas enfermé dans un cadre rigide, mais doit en quelque sorte rendre des comptes à la collectivité, représentée éventuellement par le juge du travail. Les obligations d'adaptation et de reclassement ont ensuite été reprises dans la loi. À

(39) Cons. constitutionnel, décisions du 12 janvier 2002 et du 13 janvier 2005.

(40) C'est-à-dire dans les entreprises dotées d'institutions représentatives du personnel et procédant au licenciement de onze salariés sur une période de trente jours. Il est important de rappeler que l'obligation de reclassement découlant du contrat de travail se rattache au devoir de bonne foi contractuelle de l'employeur et ne se confond pas avec les exigences relatives au reclassement du plan social, où la part du collectif est très apparente (voir notamment G. Couturier, obs. sur Soc., 15 janvier et 26 février 2003, Droit Social, 2003, 726). partir du moment où le «bloc de constitutionnalité» français (41) reconnaît simultanément le droit à l'emploi et la liberté d'entreprendre, un équilibre entre les deux doit être trouvé: dans une démocratie, ceci renvoie au rôle du juge dans l'interprétation et la mise en œuvre de la loi. Ce lien rappelé entre la justification du licenciement économique et l'obligation de reclassement est occulté par les propositions visant à confier le reclassement à des «professionnels» extérieurs à l'entreprise, et parler de "garantie de reclassement attachée à la personne» évoque un surprenant tour de passe-passe (VACHET, 2006). L'idée d'instituer une «obligation de reclassement externe » est intéressante pour les très petites entreprises, à condition de viser par ce moyen une mutualisation de la mise en œuvre du reclassement venant relayer, mais non supprimer, l'obligation de l'employeur, qui subsiste même dans une entreprise en liquidation selon la jurisprudence (42). Le thème de la flexicurité met d'autre part au premier plan l'obligation d'adaptation comme moyen de maintenir la «capacité à occuper un emploi» du salarié.

\section{Mobilités professionnelles et droits de la personne}

Le rôle du contrat de travail et le principe de bonne foi contractuelle s'avèrent également essentiels pour assurer une réalisation des mobilités professionnelles qui soit respectueuse de la sécurité des salariés. Le rapport 2005 de la Cour de cassation, chambre sociale, attire l'attention sur deux arrêts (43) ayant «clarifié les obligations des parties au contrat de travail en matière d'exercice par l'employeur de son pouvoir de direction et de refus par le salarié de l'accepter». Selon cette jurisprudence, si la mutation d'un salarié en application d'une clause de mobilité de son contrat de travail relève du pouvoir de direction, la bonne foi contractuelle exclut toutefois d'imposer à une personne en situation de «fragilité familiale» ou de santé une telle mutation. Les solutions vont dans le même sens pour un refus de modification d'horaire (44). La bonne foi contractuelle étant toutefois présumée, le salarié doit établir que le changement n'est pas voulu dans l'intérêt de l'entreprise: charge de la preuve bien lourde, qui devrait être aménagée.

(41) Le bloc de constitutionnalité, dans le contrôle des lois exercé par le Conseil constitutionnel, est constitué, outre la Constitution de 1958 et son Préambule, par le Préambule de la Constitution de 1946 et la Déclaration des droits de l'homme et du citoyen de 1789 .

(42) Sur les efforts que doit déployer le liquidateur, v. Soc., 6 mars 2007, obs. Ph. Waquet, Rev. Dr. Travail, 2007, 313. Sur le préjudice causé au salarié par un manquement de l'employeur à l'obligation d'adaptation, Soc., 23 octobre 2007.

(43) Arrêts du 27 février 2005, Bull. V. no 64.

(44) Par exemple, Soc. 15 décembre 2004, 02-44924 (charges de famille); Soc., 14 décembre 2005, 03-47721 («obligations familiales impérieuses»). 
Imaginer la suppression du contrôle du motif économique d'un licenciement en gardant le contrôle du licenciement pour faute ou motif personnel procède d'une méconnaissance tant de notre système juridique que des pratiques des entreprises. Dès lors qu'est préservé «le droit au juge» (tout de même garanti par la Convention européenne de sauvegarde des droits de l'homme et des libertés fondamentales, article 6!), les employeurs auront recours plus fréquemment au motif économique de licenciement, tandis que les salariés contesteront la nature du motif. En sens inverse, l'affaire Alcatel (prétendus licenciements pour motif personnel agrémentés de «transactions », échelonnés et permettant d'éviter un plan social) où les juges du fond ont sanctionné la fraude et ordonné quelque 170 réintégrations est une illustration tant des usages à contenu variable du motif du licenciement que du rétablissement de salariés dans leurs droits et leur dignité.

L'on ne saurait oublier aussi la théorie de l'abus de droit (sur laquelle se fonda la jurisprudence avant que ne soit exigée par la loi du 13 juillet 1973 une « cause » réelle et sérieuse de licenciement) et les cas de nullité du licenciement à raison de discriminations, d'atteinte aux libertés individuelles et collectives, qui autoriseront toujours un recours juridictionnel, ainsi que cela fut justement souligné lors du conflit sur le contrat première embauche (CPE). Ce dernier type de recours existe d'ailleurs même dans le régime du lay off at will(45)...
Quant aux ruptures «sans reproches réciproques» souhaitées par le patronat (46), il est douteux qu'elles puissent «vider la rupture de toute contestation», la jurisprudence opérant une distinction rigoureuse entre accord de rupture et transaction, et vérifiant le respect des procédures d'information-consultation en cas de départs négociés. Une rupture «d'un commun accord $»$ n'empêche pas le juge de remonter au fait générateur(47).

La volonté d'écarter tout recours au juge judiciaire paraît vouée à l'échec pour la «rupture conventionnelle» créée par l'accord national interprofessionnel du 11 janvier 2008. Comme tout juge du contrat, le Conseil de prud'hommes, s'il est saisi, appréciera la réalité du consentement des parties à la convention de rupture. Le contrôle des vices du consentement (erreur, dol) pourrait en être renforcé, sur fond de «violence économique» (48).

Des solutions assurant la protection des personnes privées d'emploi sont à imaginer, mais elles ne sauraient être novatrices sur le terrain de la sécurité de l'emploi et des parcours professionnels si elles ignorent les constructions en cours. À cet égard, toute perspective de réforme visant à améliorer la protection des personnes en matière d'emploi a pour préalable la question des critères de légitimité du licenciement et du rôle assigné au contrat de travail.

À défaut, la perspective est celle d'une restauration du pouvoir unilatéral de l'employeur.
(45) A. Mazeaud (2006, p. 591) a parlé de manière imagée de «blocs anticycloniques» pour évoquer ce qu'a d'intangible le contrôle juridictionnel en question.
(46) Selon le «projet de plan détaillé» remis par le Medef aux organisations participant à la réunion du 26 octobre 2007 sur la modernisation du marché du travail.

(47) La cour d'appel de Paris l'a rappelé le 15 mai 2007 à propos de la convention de reclassement personnalisé (Droit ouvrier, 2007.429, obs. V. Lanes).

(48) Sur «La distinction entre le consentement et la volonté», M.A. Frison-Roche, RTDC, 1995.573. Sur «le nouveau régime de la rupture conventionnelle du contrat de travail», F. Favennec-Héry, Semaine Sociale Lamy, n 1337.3. 


\section{Bibliographie}

Auer P., Gazier B., (2006), L'introuvable sécurité de l'emploi, Flammarion.

Barthélémy J., Cette G., Verkindt P.Y., (2007), «Contrat de travail, sécurisation des parcours professionnels et efficacité économique», JCP, Édition sociale, $\mathrm{n}^{\mathrm{o}}$ 7, 13 février, p. 3 (d'après le rapport remis au Conseil d'orientation pour l'emploi le 26 décembre 2006).

BARToli H., (1991), L'économie multidimensionnelle, Economica.

Bartoli H., (1996), L'économie, service de la vie, PUG.

Bonnechère M., (2007), «L'optique du travail décent», Droit ouvrier, p. 57-74.

BonNechère M., (2004), «La procédure, garantie fondamentale des droits des salariés », Droit ouvrier, p. 493.

Boulin J.-Y., Lallement M., Messenger J.-C., Michon F., (2006), Decent working time, OIT.

Bruggeman F., (2004), «Restructurations et licenciements », Droit Social, p. 852.

Cahuc P., Kramarz F., (2005), De la précarité à la mobilité; vers une sécurité sociale professionnelle, Rapport au ministre de l'Économie et de Finances, La Documentation française.

Canivet G., (2005), «L'approche économique du droit par la chambre sociale de la Cour de cassation», Droit Social, p. 951.

CAnut F., (2005), «La sanction des engagements collectifs en matière d'emploi », JCP, Édition Sociale, p. 1411.

Cerc, (2005), La sécurité de l'emploi face aux défis des transformations économiques, Rapport $\mathrm{n}^{\circ} 5$, La Documentation française.

Chauchard J.-P., (2005), «La subordination du salarié, critère du contrat de travail», in La subordination dans le travail, J.-P. Chauchard et C. Hardy-Dubernet (dir.), Cahier Travail et Emploi, La Documentation française.

Collin F., Dhoquois R., Goutierre P.H., Jeammaud A., Lyon-CAen G., Roudil. A., (1980), Le droit capitaliste du travail, PUG.

Commission européEnNe, (2006a), Livre vert. Moderniser le droit du travail pour relever les défis du XXIe siècle, Communication du 22 novembre 2006 (www.europa. $\mathrm{eu})$.

Commission européEnNe, (2006b), Promouvoir un travail décent pour tous, Communication du 24 mai 2006, COM (2006) final, (www.europa.eu).

Dockès E., (2006), «Du CNE au CPE, après jugement du Conseil de prud'hommes de Longjumeau», Droit Social, avril.

Dockès E., (2007), «Lejuge et les politiques de l'emploi », Droit Social, p. 911-916.
Duclos L., Kerbourc'h J.-Y., (2006), Organisation du marché du travail et flexibilité à la française, Document de travail du Conseil d'orientation pour l'emploi, octobre.

Eymard-Duvernay F., (2004), «Le droit du travail est-il soluble dans les incitations?», Droit Social, p. 812-815.

Eymard-Duvernay F., (2005), «Calculer ou débattre? Application aux situations de licenciement et de recrutement», in Le droit du travail confronté à l'économie, Actes de la journée d'hommage à G. Lyon-Caen, Dalloz, p. 87.

Gaudu F., (2005), «Des illusions des juristes aux illusions scientistes », in Le droit du travail confronté à l'économie, Dalloz, Thèmes et commentaires, spéc. p. 101 suiv.

Grzegorczyk C., Michaut F., Troper M. (dir.), (1993), Le positivisme juridique, LGDJ.

HÉAs F., (2000), Le reclassement du salarié en droit du travail, thèse, LGDJ.

HÉAS F., (2007), «Le droit au reclassement du salarié, en cas de restructuration de l'entreprise ou d'altération de sa santé», Droit ouvrier, p. 452.

JeAmmaud A. (dir.), (2005), Le droit du travail confronté à l'économie, Actes de la journée d'hommage à G. LyonCaen, Dalloz.

Legendre-Grandperret P., Lebon-Blanchard M.-F., Lyon-Caen P., Métin D., (2007), «Tout ce que vous voulez savoir sur le CNE sans jamais avoir osé le demander », Droit ouvrier, p. 121.

Le Goff J., (1985), Du silence à la parole. Droit du travail, société, État, 1830-1985, Éditions Calligrammes - La Digitale.

LOCHAK D.,(1990), La doctrine sous Vichyou les mésaventures du positivisme, Les usages du droit, CURAP, PUF, p. 252.

MAupain F., (2005), «Is the ILO effective in upholding workers'rights, reflections on the Myanmar experience », in $\mathrm{Ph}$. Alston, Labour rights as human tights, Oxford University Press, p. 85-142.

Mazeaud A., (2006), «Du CNE, de la flexisécurité, etc.», Droit Social, p. 591.

Morvan P., (2006), «La chimère du contrat de travail unique, la fluidité et la créativité», Droit Social, p. 959.

NADAL S., (2005), «Le destin de la négociation de branche», in La négociation collective à l'heure des révisions, G. Borenfreund, A. Lyon-Caen, M.A. Souriac, I. Vacarie (dir.), Dalloz, Thèmes et commentaires.

Pelissier J., Supiot A., Jeammaud A., (2006), Droit $d u$ travail, Dalloz, 23e édition.

Petit H., Thèvenot N. (dir.), (2006), Les nouvelles frontières du travail subordonné, La Découverte. 
Ruiz M.L., Martinez D., (2002), Les principes et droits fondamentaux au droit, OIT.

Ruiz M.L., Martinez D., (2003), «La mesure du travail décent», Revue Internationale du Travail, numéro spécial.

Romagnoli U., (2005), «Libres propos sur les rapports entre économie et droit du travail», in Le droit du travail confronté à l'économie, Actes de la journée d'hommage à G. Lyon-Caen, dir. A. Jeammaud, Dalloz.

SARGOS P., (2005) «L'horreur économique dans la relation du droit», Droit Social, 123.

Sen A., (2000), «Travail et droits», Revue Internationale du Travail, vol. 139, $\mathrm{n}^{\circ}$ 2, p. 129.

SERvais J.-M., (2004), «Politique de travail décent et mondialisation: réflexion sur une approche juridique renouvelée», Revue Internationale du Travail, vol. 143, 1-2, p. 203.

Somavia J.-A., (1999), Un travail décent, Rapport du directeur général du BIT, document OIT.

Souriac A., (1997), «Engagements et arrangements sur l'emploi: quelle efficacité juridique?», Droit Social, p. 1061.
Supiot A., (2006), «La place de la sécurité sociale dans le système des normes internationales du travail», in A. Supiot (dir.), «Protection sociale et travail décent. Nouvelles perspectives pour les normes internationales $\mathrm{du}$ travail», $\mathrm{n}^{\circ}$ spécial de la Semaine Sociale Lamy, $\mathrm{n}^{\mathrm{o}} 1272$, p. $7-12$.

Supiot A. (dir.), (1999), Au-delà de l'emploi : transformations du travail et devenir du droit du travail en Europe, Flammarion.

Troper M., (1990), La doctrine et le positivisme (à propos d'un article de Danièle Lochak), Les usages du droit, CURAP PUF, p. 287.

VAchet G., (2006), «Sécurisation des parcours professionnels: utopie ou réalité», Droit Social, 963s, séc. p. 967.

De Virville A., (2004), Pour un droit du travail plus efficace, La Documentation française.

WAQuet Ph., «Le droit du travail et l'économie à la Cour de cassation: l'exemple du licenciement économique», in Le droit du travail confronté à l'économie, Dalloz, Thèmes et commentaires, 2005, p. 115 suiv.

Wilmann C. (dir.), (2005), «Esclavage économique», Semaine Sociale Lamy, supplément no 1213 du 2 mai. 



\title{
Vers une gouvernance territoriale des risques du travail?
}

\author{
Éric Verdier(*)
}

\begin{abstract}
Partant de l'affaiblissement du statut du salarié et des mécanismes de prise en charge des risques qui peuvent l'affecter, l'auteur analyse à partir d'études de terrain des exemples d'actions collectives cherchant de nouvelles régulations. Ces tentatives ont d'original qu'elles cherchent à couvrir les risques qui pèsent sur le salarié tout en prenant en compte les enjeux propres aux employeurs. Elles tentent de mettre au point une gouvernance territoriale des risques appuyée sur des projets de développement économique et social, qui retiennent la qualité de l'emploi et du travail comme objectif principal et intègrent le souci de la compétitivité des branches ou des territoires. Elles se développent souvent en dehors des cadres habituels de la négociation sociale et professionnelle. L'auteur voit dans ces tentatives qui mêlent décentralisation, territorialisation et contractualisation, l'émergence de changements significatifs de l'action publique. Il en analyse les difficultés, liées à leur dimension cognitive, à l'engagement des acteurs et au décloisonnement de l'intervention publique qu'elles requièrent. L'article montre leur intérêt mais aussi leurs limites.
\end{abstract}

En réponse aux incertitudes et aux aléas engendrés par la relation salariale, une gestion politique des risques liés au travail s'est imposée progressivement à compter de la fin du XIX ${ }^{\mathrm{e}}$ siècle. Comme on le sait, c'est à partir du moment où les accidents liés à la réalisation du travail ont été catégorisés comme des «risques sociaux», non imputables à une responsabilité individuelle, qu'ont pu se déployer les dispositifs assurantiels caractéristiques du premier âge de l'État social (EwaLD, 1986). Tout au long du $\mathrm{XX}^{\mathrm{e}}$ siècle, ce dernier s'est développé autour d'un dispositif de protections articulant deux grandes composantes, l'une et l'autre fortement déconnectées, dans leurs principes, d'une régulation territoriale: d'un côté, l'exercice d'une solidarité nationale et interprofessionnelle qui passe par "l'institution d'un pot commun dans lequel chacun doit verser selon ses capacités et peut prélever selon ses besoins» (SUPIOT, 2005) et structuré par quatre grandes branches soigneusement séparées : maladie, famille, retraite, chômage. De l'autre, le développement d'un statut salarial qui s'accompagne d'un ensemble de sécurités attachées à la condition de travailleur dépendant, dans le cadre d'une relation

(*) Lest, Aix-en-Provence, eric.verdier@univmed.fr.

Cet article doit beaucoup au travail mené en commun avec Olivier Mériaux, notamment pour coordonner un projet de recherche, financé par le Commissariat auquel ont participé en outre Maïten Bel, Jacques Garnier, Cathel Kornig, Delphine Mercier, Aline Prévert, Francesca Sirna, Philippe Sotty, Vincent Tiano, Michel Setbon et Fred Rychen. Le rapport (versions complète et résumée) est téléchargeable à partir du lien http:// halshs.ccsd.cnrs.fr/halshs-00077806 contractuelle durable avec l'entreprise (SUPIOT, 1994 ; CASTEL, 1995). La forme juridique du « contrat de travail», en incorporant une dimension statutaire - c'est-à-dire un ensemble de garanties s'appliquant indépendamment de la volonté des parties au contrat - a permis d'inscrire la relation individuelle de travail dans un ensemble de régulations collectives offrant la «sécurité dans la subordination».

La résurgence récente de la problématique du risque appliquée à l'emploi résulte de sources d'inspiration différenciées. Les unes sont apparues ces dernières années au fil de diverses réflexions, qui, à la confluence du droit et de l'économie, s'interrogeaient sur les moyens de combiner une recherche accrue de flexibilité du marché du travail avec un renforcement de la sécurité des personnes, dans un contexte de profonde transformation des organisations productives et d'instabilité (Morin, 1997; DUPUY, LARRÉ, 1998). Les autres s'inscrivaient dans la recherche patronale d'une "refondation sociale»: s'inspirant des thèses de Beck et de Giddens, les hérauts intellectuels du Medef (EwaLD, KESSLER, 1999) s'attachèrent à faire ressortir que les systèmes classiques d'assurances sociales seraient fatalement débordés par l'extension et la fréquence des risques sociaux, du fait notamment de conduites individuelles; dès lors, il serait indispensable, éthiquement et économiquement, de transférer aux individus, et notamment aux salariés, une plus large part des responsabilités dans la couverture technique et juridique des risques encourus sur le marché du travail, notamment par une autoconstruction de son «employabilité» (investissement personnel sur la formation et ses compétences, 
acceptation de la mobilité sous toutes ses formes, engagement à retourner dans l'emploi au prix, éventuellement, d'un déclassement salarial et catégoriel temporaire...). La négociation avortée de 2001 sur la formation tout au long de la vie a fourni un exemple significatif (MÉHAUT, 2005) du débat social autour de cette problématique (1).

En tout état de cause, ces nouveaux enjeux sont symptomatiques d'un affaiblissement du statut salarial hérité des «Trente glorieuses» (CERC, 2003) à laquelle participe la remise en cause du modèle fordiste de l'entreprise intégrée: l'entreprise réseau, nouvelle figure émergente (VELTZ, 1997), articule des unités beaucoup plus mobiles depuis des rangs de sous-traitants jusque dans la grande firme ellemême, des «business units » où prévalent des projets inscrits dans des horizons beaucoup plus courts (Veltz, 2000). En sont des manifestations tangibles le développement de situations de travail hybrides entre salariat et travail indépendant (DUPUY, LARRÉ, 1998), le recours à des formes de «triangulation»" de la relation de travail (intérim notamment) ou la mise en place d'organisations productives fondées sur des réseaux d'entités économiquement subordonnées mais juridiquement indépendantes (RoRIVE, 2003). Dans tous les cas, ce mouvement se traduit par une dissociation des fonctions d'entrepreneur et d'employeur. Jointes à l'insécurité croissante de l'emploi puisque, du début des années 1980 à la fin des années 1990, la probabilité de perdre son emploi s'est sensiblement accrue quel que soit le niveau de diplôme et de qualification (MAURIN, 2002), ces évolutions affaiblissent significativement une bonne part des mécanismes institutionnels de prise en charge des divers risques susceptibles d'affecter les relations de travail. C'est alors en dernière instance l'individu qui se retrouve dans la situation de devoir, plus qu'avant, contribuer à « couvrir» ces risques.

Une alternative, qui a d'ores et déjà structuré le débat social, consiste à aller au-delà de la classique socialisation des risques pour construire plus de

(1) Ainsi l'objectif initial du Medef, lors de l'ouverture en 2001 des négociations interprofessionnelles sur la formation, était «d'introduire une plus grande part d'individualisation et de co-investissement (entreprise/salarié) dans un système où l'espace ouvert à l'individu était faible et où la formation était presque totalement prise en charge par l'entreprise» (MÉHAUT, 2005). Ceci dit, tout en étant marquées par cette approche, les propositions initiales du Medef prenaient en compte une situation initiale qui faisait reposer le poids du dispositif sur les firmes: elles prônaient la création de comptes individuels de formation mais par accord collectif d'entreprise. Il s'agissait ainsi d'articuler la négociation collective et l'engagement individuel puisque le nouveau dispositif faisait reposer directement sur le salarié une partie du coût de la formation. La formation d'une large opposition à ces propositions, allant bien au-delà de la seule partie syndicale, pour embrasser des organisations patronales de branche qui craignaient de se voir déposséder de leurs prérogatives financières, a finalement fait avorter ces projets (ibid.). sécurité dans le déroulement des parcours individuels. Le recours à la formation continue dans une perspective politique visant à renforcer l'employabilité des personnes est emblématique de la recherche tâtonnante de nouvelles régulations collectives qu'exprime, d'une manière très imparfaite (MedA, MinAULt, 2006), l'accord interprofessionnel sur la formation tout au long de la vie conclu en France en 2003.

Ce n'est pas directement à cette question de la "sécurisation" des trajectoires individuelles que s'attache cette contribution. Elle invite à s'intéresser aux formes d'action collective ( $c f$. encadré 1) qui, en amont des trajectoires individuelles, cherchent, souvent encore de manière partielle et tâtonnante, à se saisir des enjeux introduits par la croissance des risques du travail, qu'ils concernent l'emploi ou la réalisation des tâches assignées aux salariés (risques dits professionnels), en produisant de nouveaux cadres de référence. Leur premier point commun est de tenter de réunifier le traitement des risques qui peuvent toucher le salarié dans son activité de travail en allant jusqu'à prendre en considération les différents paramètres susceptibles d'affecter la compétitivité d'une branche ou d'un territoire - attractivité (d'un métier, d'un bassin d'emploi), logement, transport, etc. - et de faire retour sur la durée des relations d'emplois. De la sorte - et c'est de notre point de vue, leur force -, ces tentatives prennent aussi en compte les problèmes propres des employeurs alors qu'ils ne sont pas au centre d'approches telles que la sécurisation des parcours professionnels. Ainsi en se préoccupant de la disponibilité en quantité et en qualité des ressources humaines de nature à réduire le risque économique assumé par l'entrepreneur sur le marché des biens (voir MoRIN, 2005), ces formes d'action collective s'inscrivent - souvent explicitement - dans la perspective de promouvoir le développement économique.

Leur deuxième caractéristique commune est de construire cette intégration préventive dans un espace territorial. Une telle orientation pourrait être vue comme une manière subreptice de renouer avec un passé qui faisait du territoire le niveau privilégié du déploiement de l'assistance aux pauvres ou, plus largement, aux «classes dangereuses» (Palier, 2004). A contrario, on peut opposer que c'est dans le cadre de nouveaux processus d'intervention publique, qui entremêlent décentralisation, territorialisation et contractualisation (GAUDIN, 2003), que paraissent émerger les changements les plus significatifs de l'action publique. C'est de cette seconde perspective que part la problématique de cet article.

Par bien des aspects, cette gouvernance territoriale des risques cherche à contrebattre les comportements qui dissocient l'employeur de l'entrepreneur en s'efforçant de réintégrer les pratiques privées dans des dispositifs d'action collective. Cette réin- 


\section{Encadré 1 \\ Les terrains}

Pour chacun des cas étudiés, trois lignes d'analyse ont été retenues; chacune contribue à éclairer une facette de la coordination entre acteurs: l'institutionnalisation des dispositifs d'action par la négociation et la contractualisation; la territorialisation et, plus largement, la détermination des niveaux d'action pertinents; enfin, la construction de la connaissance et de l'expertise et leur rôle dans la coordination. Ces problématiques ont en commun de mettre l'accent sur les liens entre les résultats de l'action, les configurations d'acteurs et leur capacité à coopérer, ainsi que sur les apprentissages pratiques et cognitifs sur lesquels repose cette coopération.

Le cadre initial de cette recherche était plus large que l'objet de cet article puisque des scènes tant nationales que territoriales du dialogue social étaient prises en compte. Ainsi sept "chantiers", eux-mêmes souvent constitués de plusieurs études de cas, avaient été retenus:

1. La réforme du système de formation professionnelle: l'étude porte ici sur l'Accord national interprofessionnel (ANI) de 2003 et sa "transcription» dans la loi du 4 mai 2004 d'une part; sur la déclinaison de l'accord et de la loi dans quatre accords de branches aux caractéristiques bien contrastées (BTP, Hôtellerie-Restaurations, Assurance et Hôpitaux privés), d'autre part. Au travers des dispositifs qu'elle instaure, spécialement le droit individuel à la formation (DIF), la réforme engagée avec l'accord national interprofessionnel 2003 est symptomatique d'une sensibilité croissante des acteurs sociaux et des pouvoirs publics aux thématiques de la sécurisation des trajectoires professionnelles et de la prévention individuelle face aux risques de l'emploi.

2. Les accords tripartites de branches (ATB) en région PACA (BTP, Hôtellerie-Restauration) signés par l'État (rectorats et préfecture), les organisations professionnelles et le conseil régional. À l'initiative de ce dernier, la régulation recherchée par cette nouvelle action publique n'est plus étroitement délimitée par les enjeux propres à la formation (comme dans les contrats d'objectifs prévus par la loi de 1987) mais s'étend aux «conditions d'emploi et de travail ", voire à l'ensemble du rapport salarial. Dans les limites de l'opportunisme des acteurs économiques (par exemple recours au travail clandestin ou dissimulé) et politiques (retour intempestif de l'État central), ces nouveaux arrangements institutionnels s'efforcent d'asseoir la cohérence territoriale de l'action publique en intégrant localement des outils et dispositifs d'intervention conçus aux différents niveaux d'intervention par des acteurs publics diversifiés. Le développement de cette «gouvernance territoriale» s'est traduit par une «déhiérarchisation» des acteurs publics: l'action publique territoriale tente donc d'affirmer sa légitimité politique en construisant un intérêt collectif autour du développement économique et social.

3. Les Programmes intégrés territoriaux (PIT) de l'Agefos-PME au Lioran et à Sablé-sur-Sarthe. Ils se situent clairement dans le registre de l'initiative territoriale mais pour autant il ne s'agit pas d'un pur produit local, loin s'en faut puisque, sous de multiples aspects, cet agencement institutionnel s'inscrit dans une dynamique «multiniveaux». En particulier, elle repose sur une démarche standardisée qui, par la technicité de l'expertise, entend produire une mobilisation territoriale originale. S'appuyant sur la territorialisation croissante des politiques publiques d'emploi, de formation et de cohésion sociale ainsi que sur le développement de l'intercommunalité, l'Agefos$\mathrm{PME}$, appuyée par un cabinet d'expertise propose la réalisation d'un «Programme intégré territorial de développement de l'emploi, de la formation et des compétences". Ces politiques, souvent nommées "actions collectives", promeuvent des partenariats public-privé, dans lequel le dialogue social, la gestion paritaire et la coopération entre les pouvoirs publics et les acteurs des relations de travail sont mobilisés simultanément.

4. La Maison du travail saisonnier (MTS) du Cap-d'Agde a été créée formellement en mars 2003 et a ouvert ses portes en juillet de la même année. Elle concrétise une action publique qui traite de questions majeures pour le développement de la région Languedoc-Roussillon puisque le travail saisonnier concerne des activités économiques de premier plan pour celle-ci: le tourisme et l'agriculture. L'initiative trouve son origine dans une tentative de redéfinition locale de l'action publique en matière de travail, dont la paternité revient aux services déconcentrés du ministère du Travail. Cette approche est fondée sur un «dialogue social » indépendant des scènes classiques des relations professionnelles. Elle appelle donc une transformation des acteurs eux-mêmes au travers de nouvelles pratiques d'action. Le forum régional qui concrétise cette démarche («La Conférence régionale du travail ") a vocation à déboucher sur l'identification de problèmes d'intérêt collectif, constitutifs d'un intérêt général local. L'amélioration du travail saisonnier dans le tourisme et l'agriculture s'est ainsi imposée comme un enjeu majeur pour la «société régionale».

5. Les Groupements d'employeurs en Rhône-Alpes (GE). Un Groupement d'employeurs (GE) est constitué sous la forme d'une association (loi 1901), à but non lucratif (en rapport au délit de marchandage), avec l'objet unique de mettre ses salariés à la disposition de ses membres. Les membres d'un GE (les fondateurs et les adhérents ultérieurs) sont des personnes physiques ou morales. Les salariés sont liés au GE par un contrat de travail écrit, dans le cadre de l'application d'une même convention collective. Ils sont ensuite mis à disposition (par un contrat de mise à disposition) dans une ou plusieurs entreprises adhérentes. Le groupement est donc l'employeur de droit et détenteur du pouvoir disciplinaire vis-à-vis de ses salariés. Les entreprises utilisatrices sont par contre responsables des conditions d'exécution du travail.

6. La Ciotat, territoires en reconversion (LC). La «ville-usine» a aujourd'hui laissé place à un nouvel espace productif organisé autour de plusieurs zones productives (le site du DIAM sur l'ancien chantier naval, ATHELIA - zone d'entreprises -, le parc d'activité de Gemenos et la zone d'Aubagne) et donc de plusieurs pôles de compétences mêlant à la fois les ressources anciennes et l'arrivée d'activités nouvelles. Tous ces projets de reconversion et de réindustrialisation se conçoivent et se «négocient» à des niveaux régionaux et locaux et, notamment, au niveau de l'agglomération urbaine. 
7. Les réseaux de veille et de prévention des risques professionnels PACA en (RVP). Ils ont été mis en place en Provence-Alpes-Côte-d'Azur, ont été pensés à la fin des années 1990 et contractualisés dans le cadre du contrat de plan État-Région 2000-2006. Les RVP émanent d'une initiative locale et non de l'application d'une politique gouvernementale à décliner dans les territoires. Cet accord entre les services déconcentrés du ministère du Travail et la Région sur la prévention des risques du travail est très novateur. À travers le CPER 20002006, c'est le premier partenariat contractualisé entre la DRTEFP et la Région sur cette question des risques du travail. Pour ce faire, la DRTEFP et le conseil régional ont élaboré un appel à projets en 2000 . Ce seront vingt-trois projets qui seront retenus, traitant alors de sept thèmes différents: information et formations sur les risques, cancers professionnels, facteurs psychosociaux, évaluation des risques et système d'information, agir en entreprise. Dans ces projets collaborent des universitaires, chercheurs, médecins généralistes, médecins du travail, inspecteurs du travail, ergonomes, employeurs, CHSCT, syndicats, préventeurs, consultants.

Le principe général ayant présidé à la sélection des terrains d'investigation est celui de la confrontation entre une variété d'espaces de régulation dans lesquels sont mises en tension les formes traditionnelles d'appréhension et de prise en charge, par les partenaires sociaux et les pouvoirs publics, des risques du travail et de l'emploi.

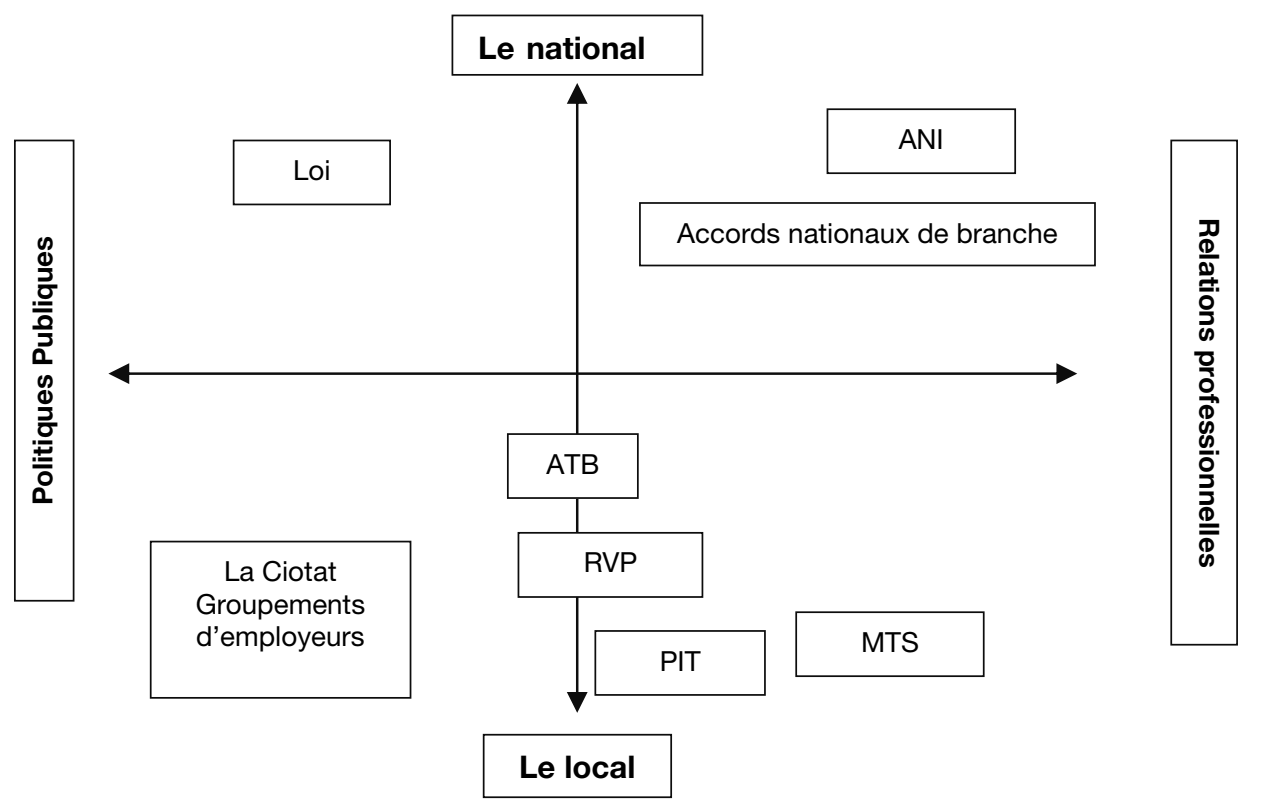

ternalisation est le plus souvent encore tâtonnante, au rythme des apprentissages organisationnels et institutionnels, ou carrément même balbutiante. Nous verrons d'abord qu'elle s'inscrit dans des projets de développement économique et social qui tentent de promouvoir un bien commun territorial. Nous montrerons ensuite que ces projets sont portés par deux types de configurations d'acteurs (voir en encadré 1, une présentation des différentes scènes étudiées). La première prolonge le sillon de la régionalisation de la formation engagée depuis vingt ans et rend compte de l'émergence, encore hypothétique, d'un gouvernement régional des compétences, de l'emploi et des qualifications. La seconde relève de gouvernances «collaboratives» de l'emploi, de la formation et du travail qui témoignent d'une déhiérarchisation des parties prenantes de l'action publique.

\section{La recherche du sens de l'action collective: comment lier développement territorial et qualité du travail?}

Dans les deux configurations évoquées à l'instant, ces projets territoriaux ( $c f$. CATLLA, 2007) s'échafaudent autour de problèmes qui articulent les questions de qualification aux enjeux du développement économique; de la sorte, s'engage une dynamique d'intéressement qui s'efforce de construire un intérêt collectif à agir, en dehors même des cadres habituels de la négociation sociale. Avec cette «nouvelle génération» de dispositifs, le champ de la régulation recherchée n'est plus étroitement délimité par les données de l'échange salarial mais s'étend aux «conditions d'emploi et de travail» prises dans un 
sens très large, allant par exemple jusqu'à prendre en compte le logement des travailleurs saisonniers ou encore les conciliations entre vie professionnelle et vie familiale. C'est à la fois une chance et une difficulté pour la prévention des risques de l'emploi et du travail: le décloisonnement des acteurs, des compétences institutionnelles et des moyens de financement d'un côté; les risques de dilution des enjeux de l'autre. Partant de questions de développement territorial croisant disponibilités des qualifications et projets économiques, ces dispositifs tendent à réintégrer dans un même référent de la qualité au travail, des objets le plus souvent disjoints par les politiques publiques et la négociation collective dans leur forme classique. Mais toute la question est de savoir si les conditions cognitives, institutionnelles et politiques sont réunies pour traiter efficacement ces enjeux.

\section{Anticiper les risques d'indisponibilité des ressources humaines adéquates aux projets de développement territoriaux}

La première étape tient à une définition partagée des problèmes d'emploi et de travail, préalable indispensable pour que l'action collective puisse avancer vers la réalisation d'un bien commun territorial qui requiert une forte coopération entre les acteurs. Dit d'une manière synthétique, l'enjeu commun aux différentes scènes étudiées est de construire, dans le cadre d'une action collective, la disponibilité d'une main-d'œuvre suffisante en nombre et en qualification en vue de favoriser la réalisation de projets entrepreneuriaux, tout en veillant à ce que leur réalisation ne soit pas excessivement dépendante d'emplois fragiles (instables ou précaires) et peu porteurs en terme de développement des compétences. Il s'agit ainsi d'être mesure de faire face, individuellement et collectivement, à d'éventuelles reconversions, résultant de pertes de compétitivité industrielle et/ou de délocalisations et, dans le même temps, de prévenir des risques économiques qui consisteraient à ne pas convertir en marchés des opportunités industrielles et commerciales.

Dans une perspective de moyen terme, les problèmes d'indisponibilité des ressources humaines se posent en terme tout autant qualitatifs que quantitatifs: il s'agit de prendre en compte des risques structurels en matière d'emploi et de travail, liés en particulier aux caractéristiques sociodémographiques du personnel actuellement employé et aux pratiques de gestion des entrepreneurs. Ainsi dans le cas de la communauté de communes de Sablé-surSarthe, les diagnostics concomitants de l'élaboration d'un projet intégré territorial promu par la communauté de communes font ressortir que les mauvaises conditions de travail subies sur le long cours par une main-d'œuvre peu qualifiée, spécialisée dans la découpe de la viande et de la volaille, pourraient se traduire, à un terme peu éloigné, par un "pic» de maladies professionnelles. Dès lors, compte tenu de la gravité déjà avérée et prévisible de ces maux, les victimes, «vieillissantes» et faiblement qualifiées sont exposées à un fort risque d'inemployabilité radicale que scelleraient des déclarations d'inaptitude qui, en cas de licenciement individuel ou collectif, engageraient probablement un processus de disqualification sociale (CENTRE AFPA, 2005). Dans un premier temps, il s'agit, pour une action collective que l'on qualifiera de «tutélaire», de préserver au mieux et au plus vite l'intégrité de personnes en danger.

Pour les employeurs, l'une des qualités de ce personnel (2) tenait à sa capacité à accepter durablement des conditions de travail pénibles, sachant que la couverture du risque pour la santé des personnes était transférée vers la collectivité et les salariés. Dès lors, les acteurs publics et privés sont confrontés à la nécessité de transformer les emplois du point de vue des qualifications et des conditions de travail, ce qui nécessite d'accélérer la réorganisation des processus de production existants et complémentairement, de diversifier les activités économiques, ce qui appelle une action durable de promotion de l'attractivité du territoire en cause.

Cette dernière orientation s'impose d'autant plus que les risques économiques d'une forte spécialisation productive territoriale, en raison du nomadisme des entreprises et/ou de délocalisations, sont accrus par leur possible connexion avec des risques «globaux» d'ordre sanitaire ou environnemental (BECK, 2001): voir l'exemple dans le cas de Sablé-sur-Sarthe, hier de la crise de la vache folle et aujourd'hui, des effets d'une propagation éventuelle de la grippe aviaire.

\section{L'émergence d'un référentiel de la qualité comme gestion préventive des risques}

L'enjeu pour l'action collective est de construire un cercle vertueux qui permettrait d'établir un «équilibre de haut niveau de qualification » (SOSKICE, 1994): l'amélioration des conditions de travail et d'emploi permet d'attirer et de fidéliser une maind'œuvre qualifiée et réactive face aux changements productifs et marchands; il en résulte une compétitivité sectorielle et territoriale accrue qui favorise une meilleure valorisation sur le plan salarial des investissements en formation et en qualification, le développement de l'emploi qualifié et l'effectivité des incitations pour les entreprises et les personnes à élever encore les qualifications.

Précisément, l'enjeu technique et politique consiste à agir simultanément sur la qualité des emplois et la disponibilité d'une main-d'œuvre

(2) La même appréciation peut être formulée à propos des saisonniers du tourisme et de l'agriculture du Cap-d'Agde, de la main-d'œuvre de l'hôtellerie des Alpes-Maritimes ou encore des saisonniers du Lioran, autres cas étudiés. 
adéquate, en jouant sur différents registres : attractivité pour susciter des offres et des demandes d'emplois de plus haut niveau, formation initiale renforcée pour structurer les qualifications, amélioration des conditions de travail et d'emploi afin de diminuer les risques de décrochage et d'abandon en cours de formation professionnelle initiale, etc. C'est sans doute l'esprit des accords tripartites de branches en PACA (ATB) dont la réussite est suspendue à la conclusion d'un échange politique entre acteurs publics régionaux et organisations professionnelles afin de construire une légitimation croisée de ces deux parties prenantes.

Dans le secteur de la viticulture languedocienne se conjuguent également plusieurs évolutions: taille croissante des exploitations, des processus de mécanisation, augmentation de la qualité attendue par les consommateurs, augmentation du contrôle et resserrement des normes sanitaires. L'extension du marché à l'exportation et l'approfondissement du marché unique ont comme conséquence des contrôles selon des normes européennes qui demandent plus de qualifications. La régulation croissante du marché des produits par les normes de qualité produit ainsi un impact certain sur le marché du travail.

Cette capacité partagée à prévenir l'incidence des risques appelle une haute qualité de coopération entre les parties prenantes des dispositifs. Sa durabilité et sa capacité à engendrer des apprentissages collectifs convertis ensuite en règles nécessitent que soit conjurée l'exposition à des risques internes à l'action publique elle-même. C'est par exemple tout l'enjeu des prestations de services apportées par la Maison du travail saisonnier dans la perspective, encore incertaine à ce stade, de canaliser les comportements opportunistes de certains employeurs: par exemple, bénéficier d'aides à la formation du personnel tout en reportant l'intégralité des risques économiques liés à la météorologie sur la seule main-d'œuvre. L'action collective, que l'on peut alors qualifier de « régulatoire», s'efforce de réduire les asymétries de ressources et de pouvoirs entre employeurs et salariés. Pour aller dans cette direction, il s'avère que délibération publique et recours à la connaissance sont des ressources pertinentes.

\section{Connaître pour débattre et coopérer}

$\mathrm{Du}$ fait de sa complexité, l'intégration des problèmes et des risques appelle des diagnostics préalables qui renforcent l'importance de la dimension cognitive de l'action publique.

Si l'on part de l'idée selon laquelle la coordination par la connaissance est essentielle à la prévention des risques, il importe d'apprécier le degré de formalisation de ce processus : les démarches et les méthodes appliquées relèvent-elles de simples échanges d'appréciations subjectives des risques, forgées par les acteurs collectifs aux prises (cas de la Maison du travail saisonnier durant ses deux premières années d'existence, ou encore du Syndicat d'aménagement de La Ciotat)? Tentent-elles d' «observer» les risques par l'entremise de diverses formes d'objectivation (traitement ad hoc de statistiques publiques, enquêtes spécifiques, etc. ), ou bien encore, de les "estimer» au moyen de modélisations théoriques (SEtBon, 2004)? La capacité d'une action collective territoriale à progresser dans l'échelle des connaissances mobilisées est l'un des critères d'appréciation de l'aptitude des acteurs, publics et privés, à réaliser des apprentissages institutionnels et organisationnels.

Ainsi, après les échanges préliminaires, les programmes intégrés territoriaux proposés par l'opérateur technique aux collectivités territoriales de Sablé-sur-Sarthe et du Lioran tentent de se situer à des niveaux sophistiqués d'appréciation des risques: les études réalisées visent à construire les risques par l'observation et mieux encore par la prévision notamment de type démographique, en vue, par exemple, de faire ressortir les expositions possibles à des difficultés de recrutement (versant économique) ou de disqualification sociale (risques encourus par les salariés).

Mais en tout état de cause, cette dimension cognitive semble difficilement dissociable de la régulation politique du dispositif. Il s'avère que ces études appellent d'autant plus la mise sur pied de forums de débat (JOBERT, 1998) pour favoriser l'appropriation des résultats que les acteurs ont initialement adopté des points de vue fortement hétérogènes. À Sablésur-Sarthe, la question des risques de disqualification d'une main-d'œuvre vieillissante et exposée à des maladies professionnelles, qui avait fait l'objet d'une enquête assez fouillée de l'Afpa, nécessitait en aval un travail de communication pour que les risques soient perçus et intégrés par les chefs d'entreprise (et en particulier par des dirigeants plus éloignés du terrain que les DRH); mais dans le cas présent, les limites sociales de cette mise en débat tiennent à l'absence des intéressés directs - les salariés - et de leurs représentants dans les forums comme dans les arènes de négociation.

Plus largement, il ressort que, dans nombre de cas, les acteurs ne disposent pas de toutes les bases de connaissances nécessaires pour élaborer les différents outils requis par une politique de prévention des risques (SETBON, 2004). Sans même évoquer les incertitudes sur les marchés des produits, il est manifeste que l'on ne détient que des informations lacunaires sur les trajectoires et les mobilités individuelles, à l'exception notable du secteur du bâtiment et travaux publics qui dispose de statistiques en la matière liées aux indemnisations consécutives aux fins de chantier. Or une incertitude scientifique portant sur les connaissances disponibles, en relation avec les conditions d'apparition de sinistres d'ampleurs variables définit un "risque de connaissance» (GÉRARD-VARET, 2001) endogène à l'action publique. 
Surmonter ou, à tout le moins, réduire le déficit de connaissances peut passer par l'expérimentation de nouvelles formules liant, à petite échelle, formations et développement des entreprises : telle semble être la démarche des accords tripartites de branche en PACA, dont la portée est conditionnée par l'aptitude à passer d'une collection de «bonnes pratiques » à l'élaboration de conventions d'applications de ces complexes échafaudages procéduraux. C'est ainsi qu'une charte locale sur la qualité de l'apprentissage (précisément dans l'hôtellerie des AlpesMaritimes) conjuguée aux résultats d'une enquête intersectorielle sur les raisons des (très nombreuses) ruptures de contrats d'apprentissage a ouvert la voie à un débat objectivé sur les causes de ce phénomène et au-delà sur l'élaboration de nouvelles règles en matière d'accueil et d'encadrement des apprentis, à l'échelle de toute la région.

Mais quelles que soient les configurations d'acteurs, la cohérence technique d'une approche intégrée des risques exige un haut niveau d'engagement dans la perspective d'articuler durablement les intérêts et les légitimités institutionnelles des parties prenantes.

\section{Vers une politique régionale du rapport salarial?}

Depuis 1994, les conseils régionaux sont en charge de l'élaboration d'un plan régional de développement de la formation professionnelle des jeunes (PRDF), étendu en 2003 à la formation des adultes. Lui préexistait depuis 1987 la possibilité pour la Région, l'État et les branches professionnelles de conclure des contrats d'objectifs territoriaux dans la perspective de rendre plus cohérente l'offre de formation. À partir de 1994, compte tenu de la faible structuration des acteurs interprofessionnels régionaux, cette démarche sectorielle est devenue un moyen d'action privilégié des conseils régionaux pour tenter de rendre effectif leur PRDF. Or cette mobilisation des intérêts par le biais des branches professionnelles a débouché au début des années 2000 sur un bilan d'ensemble assez mitigé, en PACA comme dans les autres régions. Certes, des contrats ont bien été signés en nombre. Mais leur capacité à réguler l'offre de formation d'une manière opérationnelle a lourdement peiné à s'établir (Bel et al., 2000), malgré le principe, partagé par les différents acteurs, d'une meilleure adaptation des dispositifs de formation à la demande de qualification des entreprises et des territoires. En effet, à l'instar de toutes les procédures dites «contractuelles» qui instrumentent aujourd'hui le partenariat dans l'action publique, ces documents ne créent aucune obligation mutuelle pour leurs signataires. Pis encore, ils «apparaissent davantage comme un moyen d'information réciproque qu'un outil d'harmonisation des décisions prises par les différents acteurs» (LinDEPERG, 2000). C'est sur la base d'un tel constat que la région PACA a lancé en 2003 une "nouvelle génération» de contrats d'objectifs dénommés "Accords cadres tripartites de branche» (ACTB). Fruit d'un apprentissage institutionnel, ils avancent un nouveau mode d'organisation de l'action publique contractuelle. Promouvant un intérêt régional sur les questions de formation et d'emploi, ce mode d'action s'efforce de mettre en place une régulation néocorporatiste animée par le conseil régional.

Cette tentative est nécessairement confrontée à la déclinaison territoriale du partenariat national de branche - par exemple en matière de formation tout au long de la vie - ainsi qu'aux initiatives ministérielles qui, souvent dans l'urgence, décrètent la cohésion sociale, la nécessité de la santé au travail, le développement de l'emploi, etc. Tout l'enjeu politique est pour la Région de canaliser ces initiatives «top down» pour leur donner sens et effectivité dans le cadre de sa propre stratégie territoriale. C'est dans cette perspective que la région PACA - comme d'autres - a lancé cette nouvelle génération d'accords tripartites beaucoup plus ambitieuse que la précédente au regard, tant des objets de négociation que de la structuration des acteurs et des outils mobilisés. En d'autres termes, il s'agit de construire une action collective "facilitatrice» qui, par la mise à disposition de ressources procédurales, cognitives et budgétaires (des moyens en formation) soit en position de soutenir des projets précis de développement économique.

\section{Définir de nouveaux "échanges politiques"}

Pour la Région, l'enjeu est de favoriser, par voie d'accords contractuels, une intégration des intérêts et des pratiques des acteurs publics et privés de la formation, de l'emploi et du travail dans un projet de développement régional de l'activité économique en cause. Cette perspective n'est pas sans lien avec les pratiques de gouvernement regroupées sous le classique concept de néocorporatisme (SCHMITTER, LEHMBRUCH, 1979). Afin de favoriser la mise en place d'un «gouvernement» régional néocorporatiste, les échanges politiques avec les acteurs de branche se déploient sur un double registre: le contenu des contrats, dans un souci de rationalisation de l'action publique, et les reconnaissances symboliques en vue de renforcer progressivement la légitimité des parties prenantes.

Sur le premier registre, il s'agit de «publiciser» les problèmes privés que sont les difficultés de recrutement et plus largement les enjeux sectoriels en matière de qualification et de compétences. Partant de la place décisive des activités économiques en cause pour le développement régional (BTP, HCR, Transports-Logistique, IAA), ces accords érigent la formation, l'emploi et les qualifications de ces 
secteurs en une sorte de «bien commun régional». Cette «publicisation» des activités privées doit être prise dans un double sens:

- inscrire dans le débat public la relation formationemploi et, plus largement, l'évolution du rapport salarial dans ces branches;

- favoriser la convergence des intérêts des acteurs publics et privés au sein d'un projet régional de développement économique et social.

Ainsi l'accord tripartite du BTP, conclu fin 2003, «définit les engagements pour cinq ans, de l'État, des autorités académiques et de la région ProvenceAlpes-Côte-d'Azur, ainsi que des organisations professionnelles du bâtiment et des travaux publics, pour le développement de l'emploi et de la formation dans les métiers du BTP». D'emblée, le champ est nettement plus ample que par le passé puisque tant la formation initiale et continue que la dynamique de l'emploi sont prises en compte afin «de faire évoluer tant les conditions d'exercice des métiers du BTP que la représentation partielle ou erronée qu'ont les jeunes et les adultes de ces métiers»; ainsi l'attractivité des emplois via la perception des conditions de travail par les jeunes est explicitement abordée. L'accord en cours de négociation pour les branches de la plasturgie et de la chimie va plus loin encore, puisqu'il concerne explicitement non seulement l'emploi, les compétences et la formation mais aussi l'amélioration des conditions de travail (et notamment la sécurité au travail).

Quel est le sens de l'échange politique proposé, plus ou moins clairement, par la Région? Il s'appuie sur un constat, assez largement partagé, que l'on peut formuler de la manière suivante: en réponse, notamment, aux demandes des branches qui, régulièrement, s'estimaient confrontées à des difficultés de recrutement ou même à des pénuries de maind'œuvre (transports routiers, hôtellerie et restauration, BTP), les pouvoirs publics - État et régions ont depuis 15-20 ans mis sur pied des dispositifs de formation et d'orientation des jeunes qui s'efforcent de résoudre ces problèmes. Mais une fois entrés en formation - notamment sous apprentissage -, les jeunes quittent en forte proportion les employeursformateurs $(40 \%$ d'abandons parmi les apprentis de l'hôtellerie-restauration) puis, lorsqu'ils sont éventuellement embauchés dans les emplois correspondants, les jeunes diplômés préfèrent fréquemment démissionner, compte tenu de la qualité jugée mauvaise des conditions d'emploi et de travail (cas du BTP).

Face à cette évaporation récurrente des jeunes en formation, les pouvoirs publics, la Région au premier chef, ont le sentiment d'abonder vainement ces secteurs professionnels sans que leurs responsables s'efforcent de rendre plus attractifs salaires et emplois. La contractualisation, démarche privilégiée pour régler les relations entre acteurs, est alors appelée à faire en sorte qu'aux efforts de formation des acteurs publics puissent correspondre des engagements des organisations professionnelles visant l'amélioration des conditions de travail et d'emploi. Les enjeux du travail, de l'emploi et de la formation devraient donc être traités dans une même démarche procédurale. Ce qui n'a jamais été réalisé dans l'intervention publique nationale comme dans la négociation sociale nationale.

À cet élargissement potentiel des objets des négociations tripartites, s'ajoute une dimension plus symbolique de l'échange politique. Il s'agit d'une légitimation croisée qui s'appuie sur la transaction implicite suivante: la fédération patronale du BTP accepte de soutenir la Région en vue d'insérer dans la stratégie régionale les accords conventionnels nationaux de branche ainsi que les outils d'intervention publique - que les émetteurs en soient les administrations de l'État (rectorats et direction régionale du travail), les collectivités territoriales ou la Commission européenne -, en contrepartie, avec le concours de l'État, la Région soutient financièrement, techniquement et politiquement la fédération patronale régionale afin qu'elle puisse développer des outils d'ingénierie et des dispositifs d'aide qui lui permettent de se légitimer auprès des entreprises et de s'imposer vis-à-vis des unions départementales.

\section{Un gouvernement virtuel ou le passage problématique du procédural au substantiel}

Les risques de défaillance de cette action collective sont d'ordre politique et organisationnel. Trois ans après la signature des premiers accords-cadres, l'écart peut paraître sensible entre leurs ambitions initiales et leur mise en œuvre, tel du moins qu'elle ressort des premières conventions d'application signées en juin 2005. Par exemple, alors que le déficit d'attractivité des emplois appelait les signataires à se préoccuper des conditions de travail, la mise en œuvre traite, assez classiquement, 1. d'une régulation plus serrée des cartes des formations (pour l'essentiel, CFA/lycées professionnels mais aussi, dans le cas du BTP, contrats de professionnalisation); 2. d'une intensification des actions de communication en direction des jeunes - collégiens, lycées et chômeurs - et des personnes en difficulté sur le marché du travail susceptibles de trouver dans des branches comme le BTP ou l'hôtellerie-restauration des perspectives porteuses d'insertion et de formation et 3. de la fidélisation des seuls apprentis.

C'est loin d'être négligeable, mais cela reste dans le champ classique de la formation et de l'information-orientation des publics de formés. Dans le cas du BTP, où les fédérations patronales cherchent pourtant à initier une légitimation croisée avec la Région, les employeurs ont refusé que les «fiches métiers», outils destinés à s'adresser aux jeunes, présentent les perspectives de carrières ainsi que les types de contrats et les salaires pratiqués. Le simple 
fait de donner l'information sur l'état présent conduirait ipso facto à proposer une politique pour rendre plus attractive une carrière dans la profession.

Dans un tel contexte où le nouvel échange politique se trouve bridé, certains responsables techniques de la Région auraient souhaité que l'exécutif régional adopte des positions beaucoup plus fermes et exigeantes à l'égard des employeurs, en accord avec les orientations du plan régional pour l'emploi adopté en 2005 et dans lequel s'insèrent ces accords de branches régionaux: "La négociation avec les employeurs portera sur des termes élargis tels que la valorisation et la promotion des métiers, la qualité des emplois et contrats proposés, l'amélioration de l'accueil en entreprise et la fidélisation des personnes recrutées, l'embauche des personnes en reconversion dans les secteurs qui connaissent des difficultés de recrutement, la qualification, la reconnaissance des acquis de l'expérience et l'évolution professionnelle des salariés $»$.

Adopter cette posture volontariste ne manquerait pas de bousculer quelque peu la gestion classique des rapports de la Région avec les branches fondés prioritairement, depuis le milieu des années 1990, sur la préservation d'une "paix institutionnelle», perçue comme une nécessité dans un univers institutionnel où la visibilité du conseil régional reste limitée et où les coûts de coordination sont élevés. De plus ces accords-cadres tripartites de branche peinent à dépasser le procédural, notamment parce que, au sein de chaque partie prenante - patronale et syndicale-, l'un des écueils majeurs tient à la faiblesse technique et politique des relais territoriaux censés «traduire» cette logique de branche régionalisée (voir Verdier, VION, 2005). Or la constitution de réseaux articulant les niveaux régionaux et locaux, sous la forme de communautés politiques durables, est indispensable pour espérer aller au-delà de normes générales, certes mobilisatrices initialement mais devenant, au fil du temps, de plus en plus rhétoriques. En effet, ce n'est pas qu'affaire de dispositifs pertinents mais aussi d'acteurs collectifs compétents.

\section{Une action publique ingouvernable?}

Toute la question est de savoir si les apprentissages institutionnels et organisationnels des acteurs régionaux seront suffisants pour éviter à l'action publique de rester confinée à des négociations purement procédurales et, au bout du compte, de sombrer dans une opacité croissante, à rebours des vertus supposées du «gouvernement par contrat» (GAUdin, 1999). Cette interrogation est d'autant plus fondée que le processus de déhiérarchisation des acteurs - un préfet primus inter pares dans nombre de domaines - est doublé de retours périodiques d'un État volontariste (plans de cohésion sociale, d'urgence en faveur de l'emploi... tous top down) qui engendrent de l'insécurité institutionnelle au point de fragiliser les montages élaborés patiemment au niveau régional.

En outre, la Région est d'autant plus bousculée par les injonctions nationales qu'elle ne parvient pas ou peu à jouer du caractère transversal de son action, en raison d'un mode d'organisation interne caractérisé par un cloisonnement persistant des services en charge des interventions en matière de qualifications d'un côté et d'aide aux entreprises de l'autre. L'exercice revendiqué d'une fonction de concepteur et d'animateur de l'action publique régionale en est dès lors que plus incertain. C'est à ces apories que les démarches inscrites dans des «territoires de projets » (GAUDIN, 2003) tentent d'échapper.

\section{Gouvernances collaboratives emploi-formation et projets territoriaux}

Ces initiatives résultent de l'identification conjointe d'un problème mettant en jeu l'ensemble du développement territorial: la mauvaise qualité du travail saisonnier et la compétitivité déclinante des prestations touristiques au Cap-d'Agde au cœur du plus grand ensemble balnéaire européen; une spécialisation industrielle à emplois peu qualifiés et peu attractifs à Sablé-sur-Sarthe, etc. Elles ne répondent pas d'une régulation préétablie ou cadrée par des textes législatifs mais visent d'abord à construire des dispositifs d'action efficaces; elles favorisent l'émergence d'élus locaux comme garants du bien commun territorial. Cette finalisation du dispositif vers le développement de la compétitivité territoriale grâce à un meilleur positionnement des entreprises sur leurs marchés désigne un type d'action collective que l'on peut qualifier d'entrepreneurial.

\section{Dialogue social territorial et reconfiguration du système d'acteurs}

Ces cas de gouvernance collaborative relèvent d'abord du dialogue social territorial (JoBERT, 2005), c'est-à-dire d'engagements, initialement volontaires et réversibles, qui ne débouchent pas nécessairement sur une production conjointe de règles mais plutôt sur l'élaboration de projets de développement territoriaux associant enjeux économiques et qualité $\mathrm{du}$ rapport salarial. L'enclenchement du processus s'appuie sur la mise à disposition de services opérationnels aux individus et aux entreprises, encadrés par des normes locales consignées dans des chartes ou des échanges d'engagements - plutôt que des contrats - et appuyées sur des dispositifs d'action plus ou moins formalisés.

L'initiative peut en revenir à des acteurs publics et privés de différents niveaux témoignant ainsi d'un mouvement plus large de déhiérarchisation de l'action publique (MaSSARDIER, 2003): soit des 
«entrepreneurs d'action publique» (JEANNOT, 2005), membres d'administrations déconcentrées de l'État et soucieux de favoriser la mise sur l'agenda politique territorial de problèmes structurels tels que le travail saisonnier avec la DRTEFP du LanguedocRoussillon, mais aussi des responsables d'organismes paritaires comme l'Agefos-PME avec ses programmes intégrés territoriaux qui reposent sur une ingénierie certes conçue au niveau national mais dont la démarche est directement dépendante de l'appropriation territoriale qui est en faite.

Le flou de la notion de «dialogue social» est ici, pour une fois, tout à fait apte à embrasser la très grande variété des formes de coopération territoriale multipartites échappant largement au formalisme de la négociation collective. Au fil de ces déplacements normatifs et organisationnels, ce sont les paramètres «classiques» des relations professionnelles et de l'action tripartite qui tendent à se déformer: s'y substituent des tours de table incluant administrations de l'État et des collectivités locales, élus, experts es ingénierie de projets, associations d'entreprises, etc. , sans qu'il y ait de représentativité acquise d'emblée; là où la négociation collective vise une production normative dans le cadre défini par la loi, le dialogue social territorial est avant tout l'instrument d'une action collective orientée vers la production d'un bien commun «situé» ou la résolution d'un problème localisé; le champ de la régulation recherchée s'étend aux «conditions d'emploi et de travail» prises dans un sens très large, voire aux nombreux paramètres susceptibles d'affecter la compétitivité d'un territoire: attractivité (d'un métier, d'un bassin d'emploi), logement, transport, etc. Plus l'objet de la régulation est localisé, plus la figure classique du "système» de relations professionnelles tend à s'hybrider (avec) voire à se fondre dans des instruments d'action spécifiques aux problèmes à traiter.

De fait ces constructions territoriales sont assez éloignées des procédures et des partenariats institués de la négociation sociale: par exemple, les acteurs patronaux les plus moteurs ne s'inscrivent pas nécessairement dans les organisations interprofessionnelles et sectorielles habituelles. La figure du Club d'employeurs ou du regroupement ad hoc de responsables d'entreprise est fréquente; des alliances originales entre des organisations patronales telles que l'Union professionnelle des artisans (CAPEB dans le BTP), et des syndicats de salariés autour, par exemple, d'un même référent sur le «bon» mode d'exercice professionnel peuvent être le ferment d'expériences originales. C'est souvent en opposition, du moins en distinction, vis-à-vis des fédérations dominantes et des grandes entreprises que se constituent ces configurations motrices. À l'heure d'aujourd'hui, la participation active de la représentation des salariés aux différents dispositifs est inégale, parfois faible, subordonnée à la capacité de quelques militants multipositionnés.

\section{Pragmatisme de la mise en œuvre, incertitudes sur la portée des engagements}

Ces dispositifs s'attachent à lier les réflexions et les actions des différents partenaires autour d'un objectif commun qui, dans le cas de la ville du Cap-d'Agde, est de faire d'un travail saisonnier aux conditions d'exercice sensiblement améliorées le vecteur déterminant d'un "développement économique et social durable du territoire» (Charte de la Maison du travail saisonnier). La Maison du travail saisonnier (MTS) a indéniablement engendré une dynamique locale originale articulant la construction de points de vue partagés à des services permettant d'améliorer concrètement des situations individuelles et collectives: meilleure visibilité des emplois (avant tout dans le tourisme mais aussi progressivement dans l'agriculture), ce qui favorise la rencontre offre/demande, expérimentations de solutions dans le domaine du logement, prévention de conflits prud'homaux... Désormais le thème de la sécurisation des parcours est présent dans les intentions affichées par chaque partenaire. Pour y parvenir, plusieurs actions sont énoncées mais encore assez loin de leur mise en œuvre:

- rechercher les complémentarités entre les activités saisonnières touristiques estivales et hivernales (association avec les stations de montagne);

- favoriser conjointement la professionnalisation des activités par l'accès à la formation et leur attractivité par l'amélioration des conditions d'emploi et de travail;

- introduire une pluriactivité liant tourisme et agriculture (serveur ou cuisinier, puis vendangeur, puis caviste), ce qui nécessite la mobilisation de ressources financières pour assurer les formations et convaincre les salariés que c'est possible...

En effet, au-delà de leurs représentants, les chefs d'entreprise ne sont guère engagés dans des actions visant concrètement à revaloriser les emplois, ce qui nécessiterait des engagements plus clairs en terme de garantie d'embauche pour les salariés saisonniers, en contrepartie d'aides publiques à la formation afin de les rendre plus ajustés aux besoins des entreprises. Au moment de l'enquête, l'instrumentation publique n'était pas encore parvenue à convaincre les entrepreneurs a priori bien disposés mais encore hésitants à s'inscrire pleinement dans des coconstructions relevant du moyen terme: le processus manque encore d'expérimentations probantes qui auraient pu convaincre les employeurs de modifier leurs choix en matière de qualification (voir CULPEPPER, 2003, pour une approche générale de cette question). Ainsi bien qu'inséré dans un réseau d'acteurs aux référents communs de plus en plus constitués, le dispositif territorial qu'est la MTS - comme ses autres homologues territoriaux - est-il à même de faire face à de tels enjeux dans des délais compatibles avec l'accentuation de la concurrence internationale? Or ces incertitudes sur la portée 
des engagements souscrits pourraient sérieusement limiter la capacité collective à affronter, dans la durée, des changements structurels - améliorer la qualité des produits et des emplois -, dont dépend le sens de l'action collective.

\section{Du tripartisme au "quadripartisme" ou I'implication des élus}

Le passage d'une régulation tripartite à un «quadripartisme» incluant des élus locaux traduit sans doute une tendance de fond du dialogue social territorial «élargi », à savoir la montée en puissance des intercommunalités. La rapidité avec laquelle un certain nombre d'entre elles s'installent dans le paysage des politiques publiques en faveur de l'emploi et du développement local est un symptôme supplémentaire de la structuration croissante de l'action publique par des projets fédérés autour d'un intérêt général territorial.

Cette dynamique «quadripartite», qui place en animateur central l'élu local, exprime bien la promotion des territoires et la volonté de dépasser les logiques d'action sectorielles classiques: cette prééminence d'élus, garants d'un intérêt collectif territorial, s'éloigne assez radicalement de la figure classique de la régulation croisée des années soixante (Gremion, 1976). Par exemple, la conception des programmes intégrés territoriaux (PIT) de l'AgefosPME(3), est emblématique de cette approche puisque, par-delà son abord très technique, cette formule remet en cause les formes sectorisées de l'action publique en matière de formation, incapables de répondre, selon l'Agefos-PME, aux besoins engendrés par le développement des territoires et qui nécessitent a contrario des négociations transversales (Duran, Thoenig, 1996). Elle se présente donc comme un triple remède:

- aux difficultés d'accès à la formation des PME / TPE. Le développement d'une action territoriale est dès lors apparu aux yeux de l'Agefos-PME comme le meilleur moyen de «sortir les PME de leur isolement, de favoriser les synergies entreprises/acteurs locaux, de renforcer les solidarités interentreprises en matière de formation notamment, et d'articuler les politiques professionnelles aux politiques de développement économique local» (AGEFos, 1999); - à la segmentation de l'action publique qui se traduirait par une absence de vision globale des besoins des territoires, par un cloisonnement vis-à-vis des actions en matière d'aménagement du territoire des politiques économiques relatives à l'emploi et à la création d'entreprises;

- à l'orientation des politiques de qualification. La démarche territoriale est ainsi conçue comme un contrepoids aux politiques de branche, qui produi-

(3) L'Agefos-PME est un organisme paritaire interprofessionnel collecteur des fonds de la formation (OPCA) en provenance des PME. raient des politiques de qualifications trop «sectorielles» et qui ont peu contribué à la définition des compétences transversales requises par les entreprises.

De même, dans le cadre de la Maison du travail saisonnier du Cap-d'Agde, l'objectif de «moralisation» du travail saisonnier soutenu par des contrôles renforcés du travail clandestin fait l'objet d'un consensus très large, en vue de distinguer "vrais professionnels» et «margoulins». Il s'agit de promouvoir, à terme, un référentiel d'action centré sur la qualité du travail et des prestations de services. Dans un secteur peu encadré, où la norme et la morale professionnelles sont parfois assez lâches, le dialogue social territorial renoue ainsi, sous l'égide des élus, avec la fonction première de la convention collective, l'égalisation des conditions de la concurrence. Initiatrice du projet, l'administration $\mathrm{du}$ Travail peut ainsi, en retour, rendre plus effective une politique sectorielle tournée d'abord vers les questions relatives au travail et à ses conditions d'exercice. Dans le cas des initiatives territoriales, les échanges de bons procédés sont patents. Ainsi dans le cadre d'une charte signée par les différents partenaires de la Maison du travail saisonnier, un échange implicite se met en place: lutte renforcée de la part de l'inspection du Travail contre le travail dissimulé; investissement des employeurs sur la qualité des emplois, implication des communes dans la maison de travail saisonnier et aides diverses; engagement plus important des employeurs pour le logement des saisonniers...

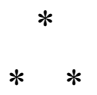

À titre de récapitulation d'une gouvernance territoriale des risques du travail, on peut identifier différents registres d'action collective définis au regard de la portée croissante de la finalité poursuivie:

- l'action collective tutélaire résulte de l'identification partagée de défaillances de la réglementation nationale destinée à assurer la protection des biens et des personnes. Risques de l'emploi et risques professionnels sont étroitement entremêlés au point de mettre en jeu la sécurité physique et psychique des personnes prisonnières de segments du marché $\mathrm{du}$ travail particulièrement pénalisants. Pour l'essentiel, l'action préventive consiste à reprendre les dispositions législatives dans des chartes signées par de multiples acteurs publics et privées dont la représentation locale des employeurs. En soi, la portée juridique de cette soft law est d'autant plus faible que préexistent de strictes obligations légales mais dont l'effectivité est depuis parfois fort longtemps douteuse. Cette démarche sourd d'une crise de légitimité des règles fondatrices de la relation d'emploi rassemblées dans le Code du travail. Reformuler les 
normes juridiques et en inscrire la mise en œuvre dans des dispositifs d'action spécifiques et partenariaux s'affiche comme un processus de réarmement de ces normes afin d'en réactiver l'effectivité et en amont la légitimité;

- l'action collective régulatoire vise à remédier aux effets négatifs, pour les individus mais aussi pour les collectivités territoriales ou professionnelles (externalités négatives liées à la mauvaise qualité des prestations et des conditions de travail), d'asymétries excessives de ressources, de pouvoirs, de connaissances, etc. ou mieux encore, à fournir aux protagonistes de la relation salariale un cadre destiné à stabiliser les engagements réciproques entre salariés et employés. Dans certains cas, cette action collective régulatoire peut emprunter différentes formes existantes de négociation sociale territoriale - commission régionale paritaire emploi-formation, commission sociale paritaire - mais s'en distingue par une pratique qui tend à embrasser des thèmes diversifiés liés à des objectifs de développement économique;

- l'action collective entrepreneuriale concerne des enjeux et des risques étroitement définis par un territoire d'action qui, autour d'un projet de développement territorial, mobilise et engage des configurations d'acteurs, dont certains sont très proches de la décision économique, dans la création de ressources spécifiques;

- l'action collective facilitatrice prend acte de deux caractéristiques majeures de l'action publique: d'une part, la nécessité d'associer, par le biais de tours de table successifs, de nombreuses parties prenantes à la construction des problèmes à traiter puis à l'élaboration de possibles projets partagés; d'autre part, l'hétérogénéité des situations à prendre en compte et, de ce fait, la nécessité de parvenir à adapter les propositions et les règles par un recours élargi à la connaissance et à l'expertise. Cette forme d'action ouvre potentiellement sur d'autres registres, notamment régulatoires ou entrepreneuriaux. Mais une telle dynamique ne s'enclenche qu'à la condition de ne pas perdre les acteurs dans des méandres bureaucratiques où la procéduralisation deviendrait une fin en soi (DE MunCK, 2002).

Certes à court terme, paraît encore improbable l'émergence d'un référentiel commun (JOBERT, Muller, 1987) sur le «développement durable du travail» dans ses différents volets - intégrité des personnes, qualifications, rémunérations et promotion - et dans lequel les questions de santétravail prendraient une place éminente et reconnue. Néanmoins, malgré leur fragilité et leur réversibilité, les différents dispositifs étudiés ne témoignentils pas d'une tendance à décloisonner les objets classiques de l'intervention publique en matière de formation professionnelle, de travail et d'emploi? C'est, semble-t-il, d'autant plus le cas lorsque se met en place une dynamique de création endogène de ressources économiques et humaines allant audelà d'un processus assuranciel de couverture des risques du travail.

\section{Bibliographie}

Agefos-PME, (1999), Territoires et projets. Pour une politique territoriale et interprofessionnelle de développement de l'emploi et de la formation dans les petites et moyennes entreprises, rapport Miméo, Paris.

BECK U., (2001), La société du risque. Sur la voie d'une autre modernité, Aubier.

Bel M., Gérard-Varet L.-A., Verdier E., (2000), "L'évolution des modes de construction de l'offre de formation professionnelle» (avec) in Comité de coordination (ed.), Évaluation des politiques régionales de formation professionnelle 1997-1999, Tome 1, Paris, La Documentation française, p. 183-215.

Catlla M., (2007), «De la genèse d'une régulation territorialisée à l'émergence d'une gouvernance territoriale», in Pasquier R., Simoulin V., Weisbein J. (dir.), La gouvernance territoriale, Paris, LGDJ, Tome 44, coll. «Droit et Société», p. 89-107.

Castel R., (1995), Les métamorphoses de la question sociale, une chronique du salariat, Paris, Fayard.

Centre AfPa, (2005), Âge et travail en pays de la vallée de la Sarthe, diagnostic de la situation d'emploi des actifs de plus de 45 ans, Miméo Afpa, Le Mans, 72 p.

CERC, (2003), «Les risques liés à la transformation de l'emploi», Les Papiers du Cerc, n 2003-03.

Culpepper P., (2003), Creating Cooperation, How States Develop Human Capital in Europe, Cornell University Press, Ithaca and London.

De Munck J., (1997), «Normes et procédures: les coordonnées d'un débat», in De Munck J., Verhoeven M., Les mutations du rapport à la norme, Bruxelles, De Boeck, p. 25-63.

DUPUY Y., LARRÉ F., (1998), «Entre salariat et travail indépendant: les formes hybrides de mobilisation du travail», Travail et Emploi, $\mathrm{n}^{0}$ 77, p. 1-13.

Duran P., Thoenig J.-C., (1996), «L'État et la gestion 
publique territoriale», Revue Française de Science Politique, vol. 46, n 4, p. 580-623.

Ewald F., (1986), Histoire de l'État-Providence, Paris, Grasset.

Ewald F., Kessler D., (1999), «Les noces du risque et de la politique», Le Débat, p. 55-72.

Gaudin J.-P., (1999), Gouverner par contrat, Paris, Presses de Sciences Po.

Gaudin J.-P., (2003), L'action publique. Sociologie et politique, coll. «Amphi», Presses de Sciences Po et Dalloz, Paris.

Gérard-Varet L.-A., Lazzeri Y., Rychen F., (2002), «Risques locaux et action collective: éléments pour une problématique», in Pivot C., Rychen F., Gérer les risques collectifs (sous la direction de), Éditions de l'Aube.

Gremion P.,(1976), Le pouvoir périphérique. Bureaucrates et notables dans le système politique français, Paris, Le Seuil.

JEANNOT G., (2005), Les métiers flous: travail et action publique, coll. «Travail et action humaine», Octarès.

JoBERT A. (coord.), (2005), Les nouveaux cadres du dialogue social: l'espace européen et les territoires, rapport pour le Commissariat général du Plan, Miméo.

Jobert B., (1998), «La régulation politique: le point de vue d'un politiste», in Commaille J., Jobert B. (ed.), Les métamorphoses de la régulation politique, LGDJ, 24, Paris, p. 119-146.

Jobert B., Muller P., (1987), L'État en action. Politiques publiques et corporatismes, PUF, Paris.

LINDEPERG G., (2000), Les acteurs de la formation professionnelle: pour une nouvelle donne: rapport au Premier ministre, Paris, La Documentation française, Collection des rapports officiels.

Massardier G., (2003), Politiques et actions publiques, Science Politique U, Armand Colin, Paris.

Méda D., Minault B., (2006), «La sécurisation des trajectoires professionnelles», Dossiers, ${ }^{\circ}$ 107, Dares, Ministère du Travail et des Affaires sociales.

MÉHaut Ph., (2005), «Reforming the training system in France», Industrial Relations Journal, 36: 4, p. $303-$ 317.

MÉRIAUX O., Verdier E., (2006), Les relations professionnelles et l'action publique face aux risques du travail et de l'emploi, Recherche réalisée pour le Commissariat général du Plan, Miméo, Paris.
Morin M.-L., (1997), «Réseaux de production localisés et relations de travail», Note LIRHE 239, avril 1997, Toulouse.

Morin M.-L., (2005), «Le droit du travail face aux nouvelles formes d'organisation des entreprises », Revue Internationale du Travail, vol. 144/1, p. 5-30.

PAlier B., (2004), Gouverner la sécurité sociale, coll. «Essais Débats, Quadrige», PUF, Paris.

Rorive B., (2003), «Qui porte la responsabilité sociale dans l'entreprise réseau?», Communication du Lentic (Université de Liège) pour le projet Relier à la $5^{\mathrm{e}}$ université de printemps de l'audit social IAE de Corse, 2223 mai.

Rychen F., Pivot A. (dir.), (2002), Gérer les risques collectifs, Éditions de l'Aube.

Schmitter Ph., Lehmbruch G. (ed.), (1979), Trends toward Corporatist Intermediation, Beverly Hills (CA), Sage.

SEtbon M., (2004), Risques, sécurité sanitaire et processus de décision, coll. «Médecine des risques », Elsevier, Paris.

Soskice D., (1994), «Reconciling Markets and Institution: The German Apprenticeship System» in Lynch Lisa (ed.), Training and the Private Sector; International Comparisons, The University of Chicago Press, Chicago, p. 25-60.

Supiot A., (1994), Critique du droit du travail, Paris, PUF.

Supiot A., (2005), Homo Juridicus. Essai sur la fonction anthropologique du droit, coll. «La couleur des idées», Seuil, Paris.

Veltz P., (1997), Mondialisation, villes et territoires: l'économie d'archipel, coll. «Économie en liberté», PUF, Paris.

Veltz P., (2000), Le nouveau monde industriel, Gallimard, Paris.

VERdier E., Vion A., (2005), «Mix of contracts or contractual mix-up? The coordination of public intervention on access to work in France», International Journal of Public Policy, n 1-2, p. 161-184. 